Revue des patrimoines

Le cheval et ses patrimoines (1ère partie)

\title{
Le cheval de travail dans les fonds d'entreprises des Archives nationales du monde du travail
}

Gersende Piernas

\section{(2) OpenEdition}

Journals

Édition électronique

URL : http://journals.openedition.org/insitu/9644

DOI : 10.4000/insitu.9644

ISSN : 1630-7305

Éditeur

Ministère de la culture

Référence électronique

Gersende Piernas, «Le cheval de travail dans les fonds d'entreprises des Archives nationales du monde du travail », In Situ [En ligne], 18 | 2012, mis en ligne le 31 juillet 2012, consulté le 01 mai 2019. URL : http://journals.openedition.org/insitu/9644; DOI : 10.4000/insitu.9644

Ce document a été généré automatiquement le 1 mai 2019.

\section{(c) $($ i) $(9)$}

In Situ Revues des patrimoines est mis à disposition selon les termes de la licence Creative Commons Attribution - Pas d'Utilisation Commerciale - Pas de Modification 4.0 International. 


\title{
Le cheval de travail dans les fonds d'entreprises des Archives nationales du monde du travail
}

\author{
Gersende Piernas
}

1 Les Archives nationales du monde du travail ${ }^{1}$ collectent depuis 1993 les archives issues de nombreux domaines d'activités et en provenance des différents acteurs du travail, à savoir de l'entreprise au syndicat, en passant par les comités d'entreprises et le mouvement associatif. Les archives d'entreprises représentent environ $80 \%$ de cette collecte, qui résulte de versements pour les archives publiques et de dons, dépôts révocables, achats, séquestres, déshérences pour les archives privées. La collecte des archives privées s'effectue sur sollicitation de dirigeants mais aussi d'anciens employés, du cadre à l'ouvrier. Ainsi, ces archives sur multiples supports (papiers, plaques de verre, maquettes, audiovisuels) proviennent-elles de toute la France avec néanmoins une forte présence du Nord-Pas-de-Calais. La collecte privilégie les fonds à envergure nationale voire internationale mais surtout complets, c'est-à-dire tous les documents qui reflètent l'ensemble des activités de l'entreprise comme le montre le plan type de classement suivant: constitution de l'affaire, administration générale, patrimoine, service juridique, activités financières, comptabilité, personnel, techniques et production, activité commerciale et distribution.

2 En effet, une entreprise, ce n'est pas seulement des locaux, des outils et des produits finis mais aussi des acteurs vivants : à savoir des hommes et des animaux, et notamment des chevaux qui produisent de l'énergie, qui tractent, déplacent et transportent. C'est le cheval de travail (on exclut le cheval de courses ou de sport, le cheval policier ou militaire), ou «cheval moteur » comme l'appelle Daniel Roche ${ }^{2}$. Ainsi, parmi ces fonds conservés aux ANMT, trois traitent directement du cheval :

3 - Fonds Dailly, maîtres des postes aux chevaux à Paris, 19 AQ (don, 1945)

4 - Fonds de la sellerie bourrellerie Legrand (Amiens), 178 AQ (achat, 1982) 

"cheval» n'apparaît pas nécessairement dans lesÀ inventaires car les archivistes Ainsi, le cheval n'est que très peu remarqué et décrit dans les fonds d'entreprises, pour lesquels on s'attache davantage à mettre en valeur l'historique, le personnel et la production. On trouve d'autres termes relatifs au cheval, des adjectifs surtout (« équin », " hippique ", " hippomobile», etc.), mais aussi des noms communs (étalon, hongre, jument, poulain, palefrenier, etc.). Cependant dans de nombreux inventaires, on ne relève aucune mention alors que le fonds regorge de documents évoquant le cheval de travail. Il convient donc de dépouiller les fonds. Il est proposé ici de découvrir ces documents trouvés à partir des fonds suivants: Maîtres des postes Dailly, Sellerie bourrellerie Legrand, Compagnie du chemin de fer du Nord, Compagnies minières de charbon (Anzin, Béthune, Bruay, Carvin), Charbonnages de France, BTP (Chagnaud, Pelnard-ConsidèreCaquot), Établissements de construction mécanique agricole Savary, Brasserie Delahaye, René Courtois/Colonie agricole de Saint-Maurice.

8 On retrouve donc ces documents aux typologies variées dans les différentes parties des fonds consultés à savoir : l'administration, la gestion, le personnel, puis les activités. C'est dans cet ordre que nous les évoquerons.

\section{Le cheval, élément constitutif des entreprises}

\section{Un acteur parmi d'autres pour l'administration générale}

Les actes constitutifs d'une entreprise, statuts et actes de sociétés, exposent les raisons, les objectifs et les moyens de celles-ci. Le cheval y est évoqué en tant que moyen de transport ou de production d'énergie. Parmi ces actes, on trouve d'autres documents comme les projets (fig. $\mathbf{n}^{\circ} \mathbf{1}$ ) alliant le cheval avec une autre technologie, le chemin de fer ou, à l'inverse, comme les manifestes ${ }^{5}$ de défense du cheval rédigés par des entreprises qui luttent contre l'arrivée et le développement du chemin de fer. 


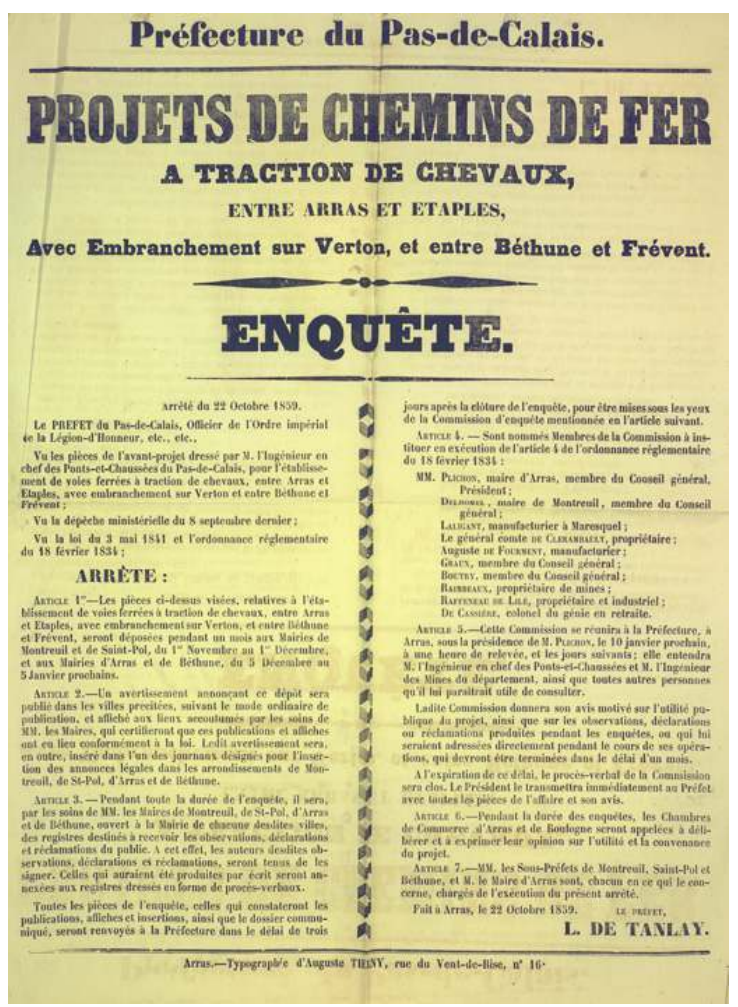

Affiche de projets de chemins de fer à traction de chevaux entre Arras et Étaples, 1859.

(C) ANMT, Compagnie du chemin de fer du Nord, 202 AQ 2043.

Les textes règlementaires internes et les textes législatifs, dont une copie est souvent conservée dans les fonds, donnent la place, l'emploi et les règles de conduite des chevaux et des machines (véhicules, outils, etc.) à la fois dans l'entreprise mais aussi à l'extérieur en cas de déplacement sur la voie publique ce qui est le cas de la Poste aux chevaux. Ses déplacements dans Paris sont régis ${ }^{6}$ par la Préfecture de police de Paris au travers de l'«Ordonnance concernant la conduite des chevaux et des voitures dans Paris", du 26 août 1861. On distingue aussi la place du cheval avec ses droits et ses obligations dans le Recueil de lois sur le roulage et le transport de 1806, et le Code des postes et relais de France depuis 1789 de 1839. Le Code des chemins de fer et des postes en France de 1841, entre autres, montre l'articulation nécessaire et fragile entre le cheval, la route et le chemin de fer.

Les organes de décisions et de contrôle (direction, assemblées générales, actionnaires, gérants, etc.) sécrètent des procès-verbaux, des circulaires et des notes mettant en application les textes règlementaires. Afin d'exercer leur contrôle soit les uns par rapport aux autres, soit sur d'autres rouages subalternes de l'entreprise, ils reçoivent de nombreux rapports et bilans très riches en informations et statistiques annuelles sur tous les aspects de l'entreprise, dont ceux relatifs aux chevaux et aux machines qui leur sont liées : nombres, journées de travail, gains et pertes. Au sein de la Compagnie générale des omnibus de Paris, une comparaison est effectuée en 1879 entre les lignes avec chevaux et lignes sur rail avec des tableaux très complexes ${ }^{7}$. Les rapports sur les opérations $d u$ service de la cavalerie et des fourrages de la Compagnie générale des omnibus de Paris présentés au conseil d'administration ${ }^{8}$ font entrer dans la gestion même des écuries: 
nombre de chevaux, sexe (entiers, hongres, juments ou poulains), provenance, race, couleur de robe, âge, raisons des décès et des réformes, alimentation et soins.

\section{Un élément du patrimoine de l'entreprise}

Le cheval fait ainsi partie du patrimoine vivant et matériel de l'entreprise comme le montrent les archives du capital immobilier et mobilier de l'entreprise. Les estimations et les bilans annuels incluent les chevaux dans les biens de l'entreprise avec leur valeur réelle à l'achat et à la vente ultérieure. Ils sont à la fois dans l'actif pour leur valeur et dans le passif en cas de non paiement de leur prix d'achat.

Figure 2

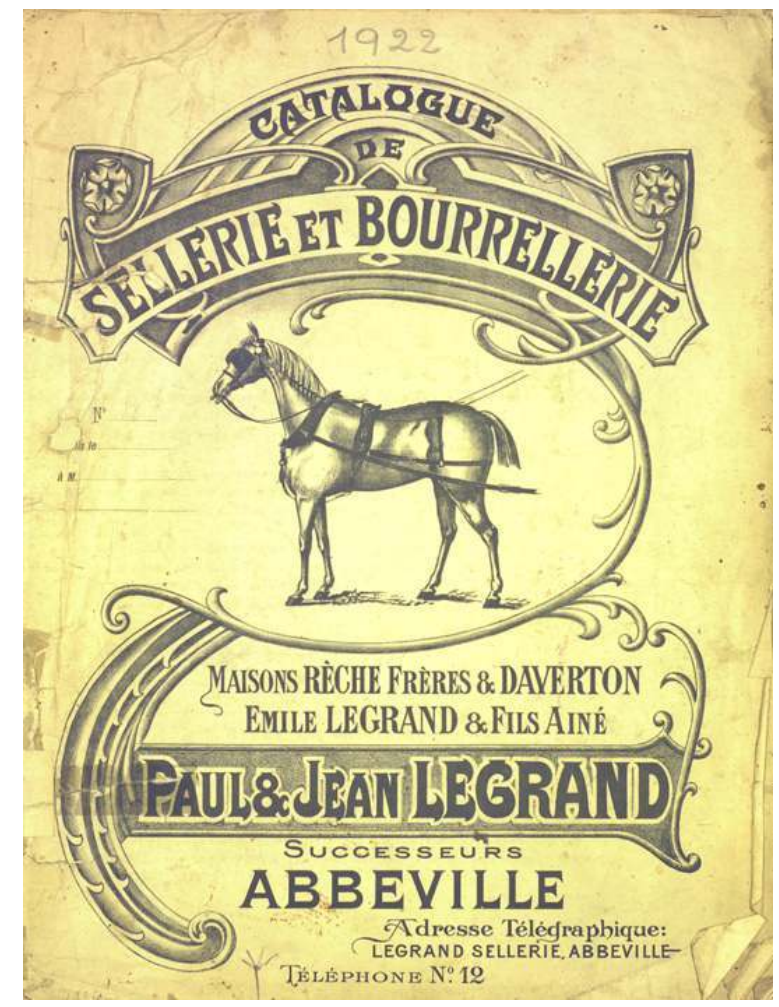

Catalogue de vente de la sellerie-bourrellerie Paul \& Jean Legrand à Abbeville (Somme), 1922

(c) ANMT, Sellerie-bourrellerie Legrand, 178 AQ 001.

On y trouve aussi la mention du matériel nécessaire à leur maniement et à leur travail (machines, voitures, véhicules, wagonnets, etc.). Cet équipement se caractérise par les articles de sellerie-bourrellerie: brides, mors, colliers, selles, etc. On retrouve des catalogues de fournisseurs mais également de fabricants comme dans le fonds de la sellerie-bourrellerie Legrand à Abbeville dans la Somme (fig. $\mathbf{n}^{\circ}$ 2). Le matériel de travail qui permet de produire de l'énergie est également représenté, telle cette roue horizontale à la Compagnie des mines d'Anzin, à laquelle sont attelés deux chevaux, qui, en tournant, font remonter par un système de poulies les seaux remplis de charbon des profondeurs du puits (fig. $\mathbf{n}^{\circ} \mathbf{3}$ ). Les fonds de sociétés de construction mécanique agricole ou électrique contiennent aussi des documents sur les autres types de véhicules tractés par les chevaux. Dans le fonds Thomson-SFR, on peut consulter plusieurs plans-calques de postes de TSF 
hippomobiles datant des années 1910-1920. Des plans-calques de houes à cheval et autres machines agricoles sont conservés dans le fonds des Établissements Savary.

Figure 3

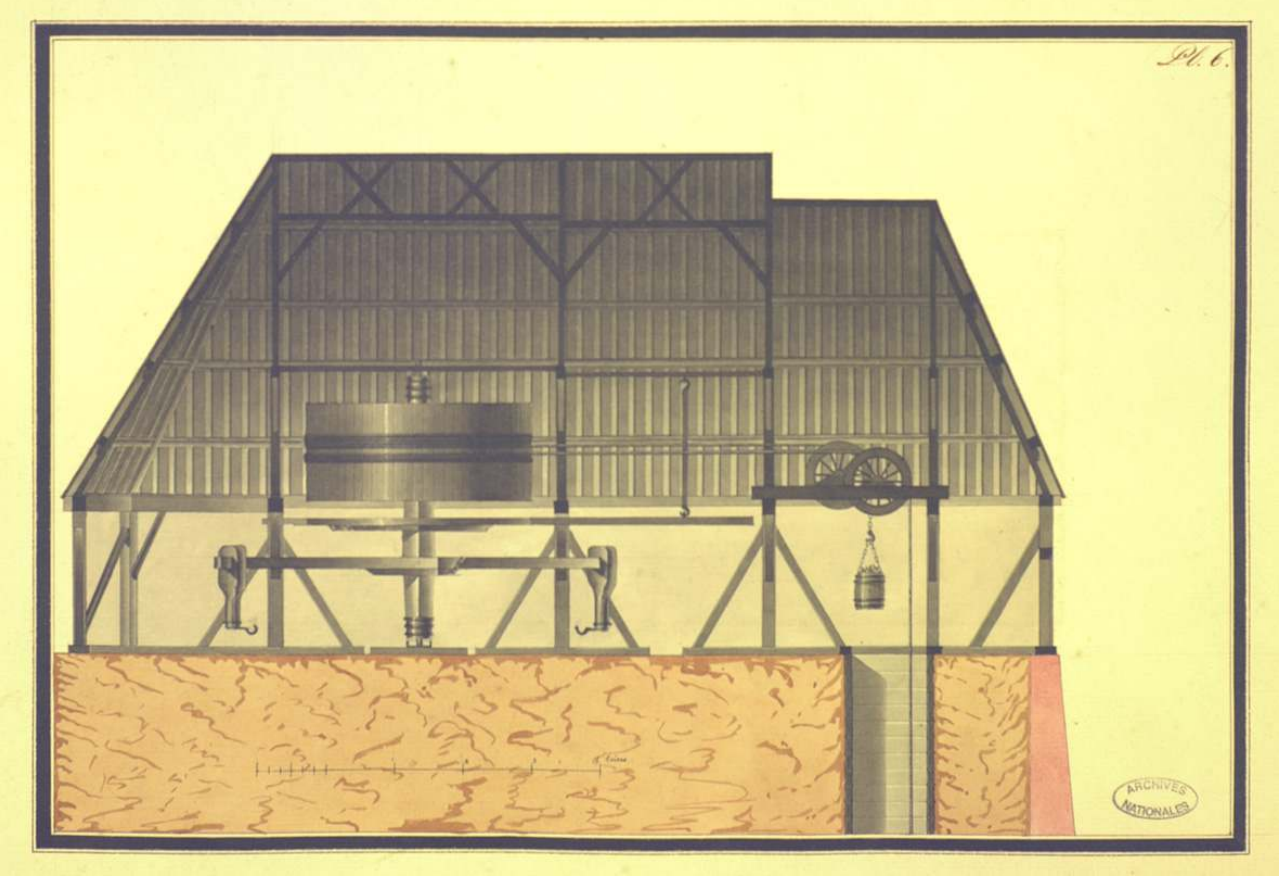

Puits à hipporemontée, planche $n^{\circ} 6$ de l'ouvrage aquarellé intitulé « Machines et outils à l'usage dans les mines d'Anzin », [XIXe siècle]

(c) ANMT, Fonds Compagnie des mines d'Anzin, 49 AQ 16.

Les chevaux, comme leur équipement et le personnel qui s'en occupe, nécessitent des locaux spécifiques : écuries, remises, manèges, bureaux, logements, etc. Ces locaux font partie du patrimoine immobilier des entreprises qui en dressent les plans ainsi que des règlements ou des notes d'utilisation. Il s'agit de bâtiments fixes, comme les écuries impressionnantes de la Compagnie générale des omnibus dans le XVIIIe arrondissement de Paris pouvant contenir jusqu'à 400 chevaux sur plusieurs niveaux, dans des salles de 12 à 42 box, équipées de cours, de greniers, de magasins, de réservoirs d'eau, de hangars, de bureaux et de lieux de stockage du fumier (fig. $\mathbf{n}^{\circ} \mathbf{4}$ ). Elles sont conçues pour gagner de l'espace, du temps et surtout minimiser les nuisances pour le voisinage. Le plan des établissements et des écuries de la Poste aux chevaux à Paris, à l'angle de la rue de Pigalle et de la rue de la Tour des Dames, est moins détaillé mais montre un enchevêtrement de bureaux, d'écuries, de cours et de jardins tout aussi vaste. On note aussi la présence d'écuries dans les dépôts et les gares des grandes compagnies de chemin de fer à Paris. En effet, elles ont recours aux chevaux pour transborder les marchandises, déplacer le matériel ferroviaire, ou relier leurs différents dépôts dans la capitale et sa proche banlieue. 
Figure 4

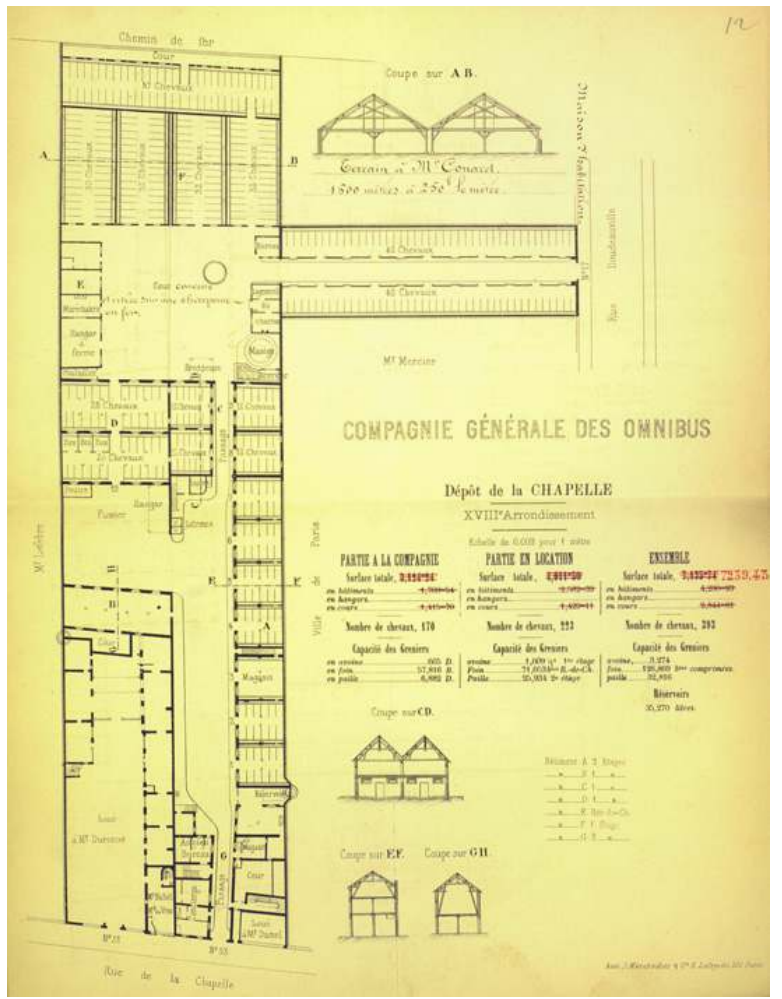

Plan du dépôt de la Chapelle de la Compagnie générale des omnibus à Paris, XIXe siècle. (c) ANMT, Compagnie du chemin de fer du Nord, 202 AQ 1635.

15 À côté de ces écuries bien équipées, on peut évoquer le cas des chevaux de mine qui disposent d'un espace certes fixe et en dur mais très rudimentaire et exigu, soit bien souvent une simple stalle en bois avec un espace contre le mur pour le fourrage (fig. $\mathbf{n}^{\circ} 5$ ). 
Figure 5

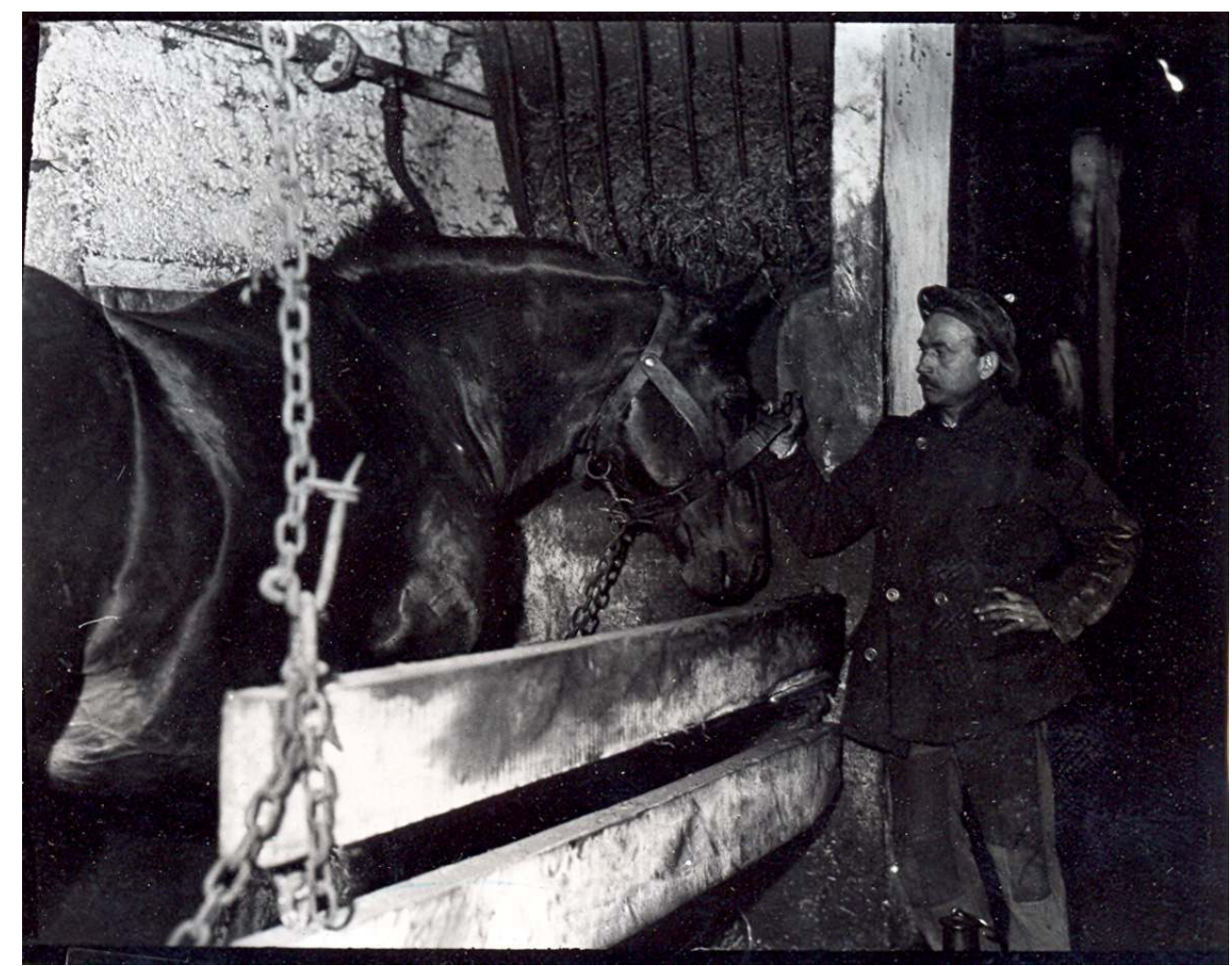

Cheval et son palefrenier dans une stalle en bois d'une mine du Nord-Pas-de-Calais, Collection Le Boyer, [1937-1945].

(c) ANMT, Charbonnages de France, 20070084054.

16 Si le cheval de mine ne remonte que mort à la surface une fois son temps de service écoulé, celui des postes, des omnibus et des chemins de fer peut se refaire à la campagne où ces entreprises possèdent des terrains à cet effet. Les compagnies de chemin de fer ont même développé des écuries mobiles, constituées de wagons de 2 à 6 box au début du XX siècle, pour leur usage mais aussi pour le routage des chevaux à vendre et pour le transport rapide des chevaux militaires à transporter rapidement d'un point à l'autre du territoire (fig. $\left.\mathbf{n}^{\circ} \mathbf{6}\right)$. 
Figure 6

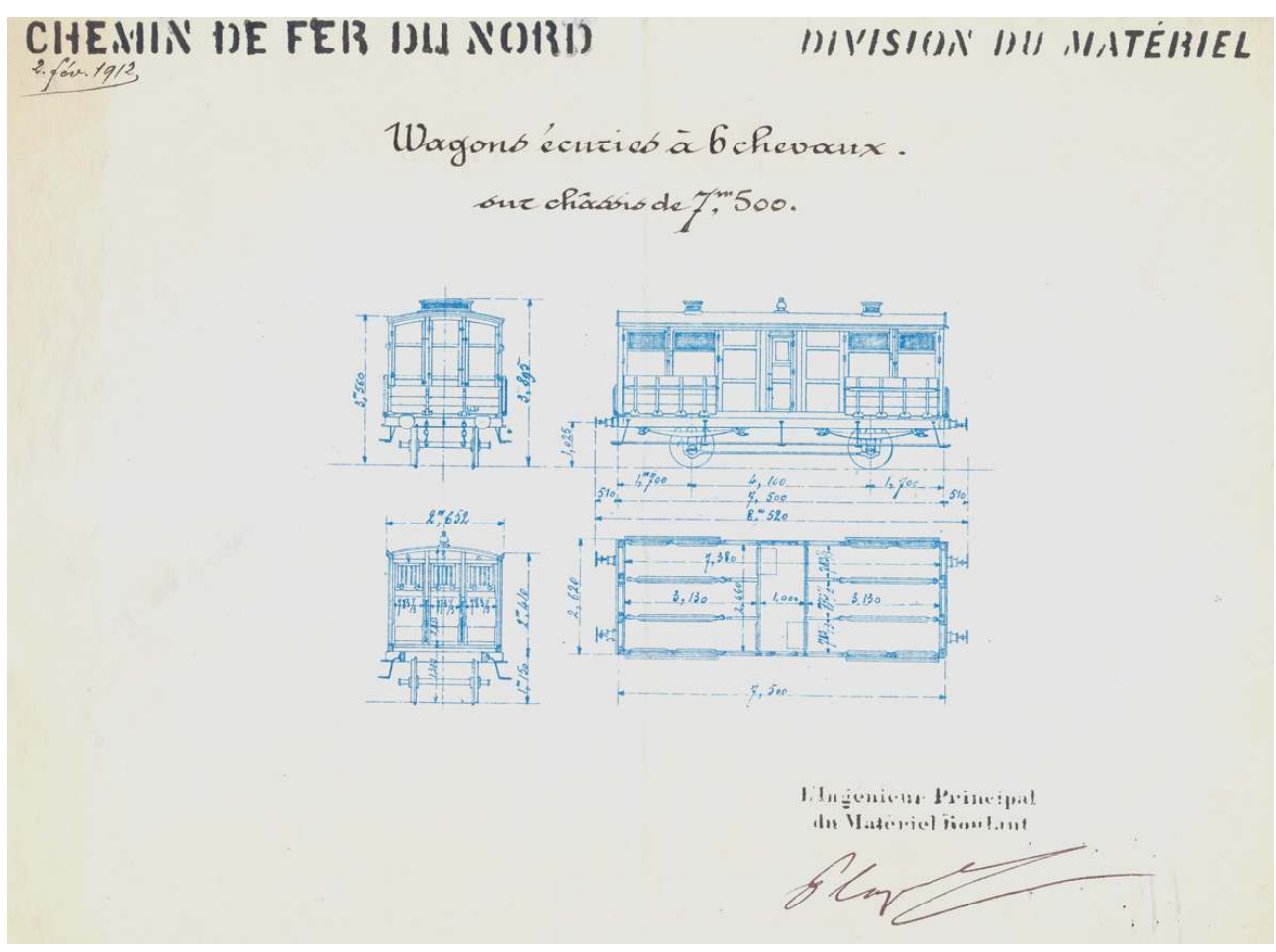

Wagons écuries de la Compagnie du chemin de fer du Nord, 1912.

(C) ANMT, Compagnie du chemin de fer du Nord, 202 AQ 1293.

\section{Gérer le cheval : entre rentabilité et risques}

\section{Le cheval, unité comptable}

Les archives comptables incluent les dépenses et les recettes relatives à l'achat, l'entretien, le soin et l'activité des chevaux. Elles prennent la forme de registres, tableaux, notes de calculs, entre autres, journaliers, hebdomadaires, mensuels et annuels. Dans le cas de la Compagnie générale des omnibus, les tableaux mentionnent, en fonction de la nature des omnibus et des tramways, les recettes comme suit: le nombre moyen des voitures, le nombre de journées de chevaux, la différence par rapport à la référence (jour, semaine, mois ou année), les recettes totales et la recette par journée de voiture et par journée de cheval. Ces recettes sont ensuite rapportées aux dépenses (fig. $\mathbf{n}^{\circ} \mathbf{7}$ ). L'alimentation et le fourrage des chevaux sont aussi comptabilisés : le prix et la quantité des denrées (grains, foin, paille) entrent dans ces mêmes tableaux. On note ainsi l'apparition de mesures comptables liées au cheval, à ses accessoires et à ses capacités. S'en dégage le prix de revient d'un poste de cheval calculé en juillet 1924 par la Compagnie des mines de Carvin (fig. $\left.\mathbf{n}^{\circ} \mathbf{8}\right)$. Il apparaît très vite dans les entreprises que l'achat d'automobiles, de camions et d'autres machines permettent de remplacer avantageusement le cheval qui se voit progressivement supplanté dans les années 1930 puis définitivement dans les années 1960. 
Figure 7

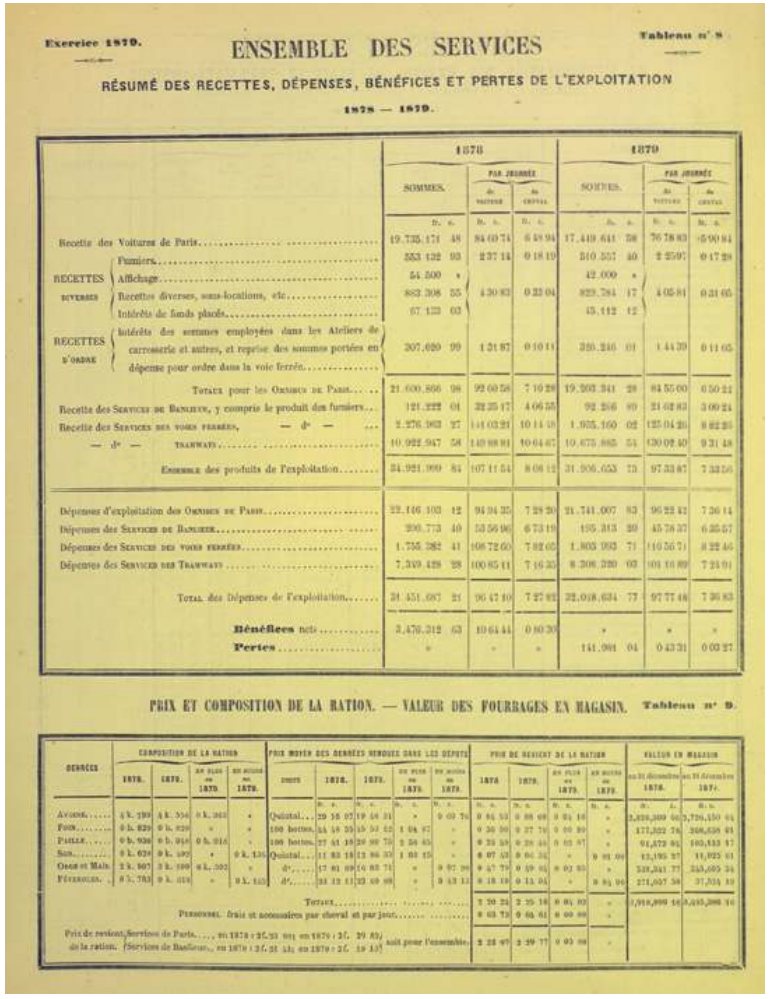

Tableau annuel des recettes et dépenses de la Compagnie générale des omnibus de Paris, 1878-1879. (c) ANMT, Maîtres des postes Dailly, 19 AQ 38. 


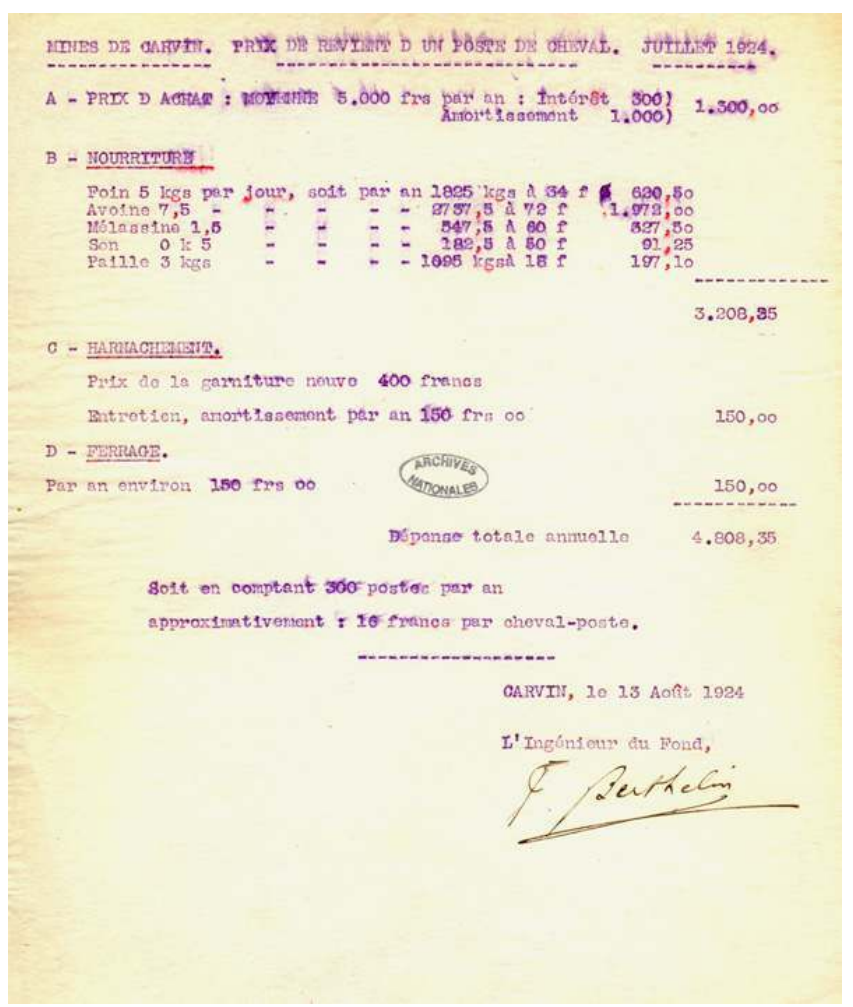

Note sur le prix de revient d'un poste de cheval dans les Mines de Carvin, juillet 1924.

(c) ANMT, Compagnie des mines de Carvin, 1994057054

\section{Un acteur soumis aux risques pour le service juridique}

Le cheval, ses équipements et ses écuries sont une partie du patrimoine d'une entreprise qui s'avère stratégique en cas de conflits et ce jusqu'à la Seconde Guerre mondiale. C'est pourquoi ils font l'objet de recensement par les mairies en vue de réquisitions en cas de conflit armé. Les entreprises déclarent ainsi leurs chevaux et leur matériel et reçoivent en échange des certificats de déclaration et des listes de chevaux mobilisables à partir de telle date en fonction de leur sexe, race, ou âge. Certains, trop âgés, blessés ou trop petits, sont d'emblée réformés. Si ces entreprises subissent des dommages ou des pertes lors des conflits, elles peuvent prétendre auprès des autorités à des dommages de guerre (fig. $\mathbf{n}^{\circ} \mathbf{9}$ ) . L'élaboration de leur dossier de demande par leur service juridique les amène à dresser la liste des chevaux blessés, perdus ou tués ainsi que le matériel détruit avec leur valeur initiale. Dans le Nord, les compagnies minières ont été la cible de bombardements allemands pendant la Première Guerre mondiale et ont perdu alors en surface de nombreux chevaux et leurs installations ont été détruites par les obus, événements mentionnés dans les rapports dits « Dates de bombardements par canons ", où hommes et chevaux se côtoient dans la mort. Pour ces derniers, nous disposons même de leur nom, de la date et du prix de leur achat, de leur valeur avant la Guerre ainsi que de leur valeur de remplacement émise à partir de la soustraction entre le prix d'achat et la valeur en 1914 (fig. $\mathbf{n}^{\circ} \mathbf{1 0}$ ). Il en est de même pour les harnais et les voitures endommagés ou détruits. 
Figure 9

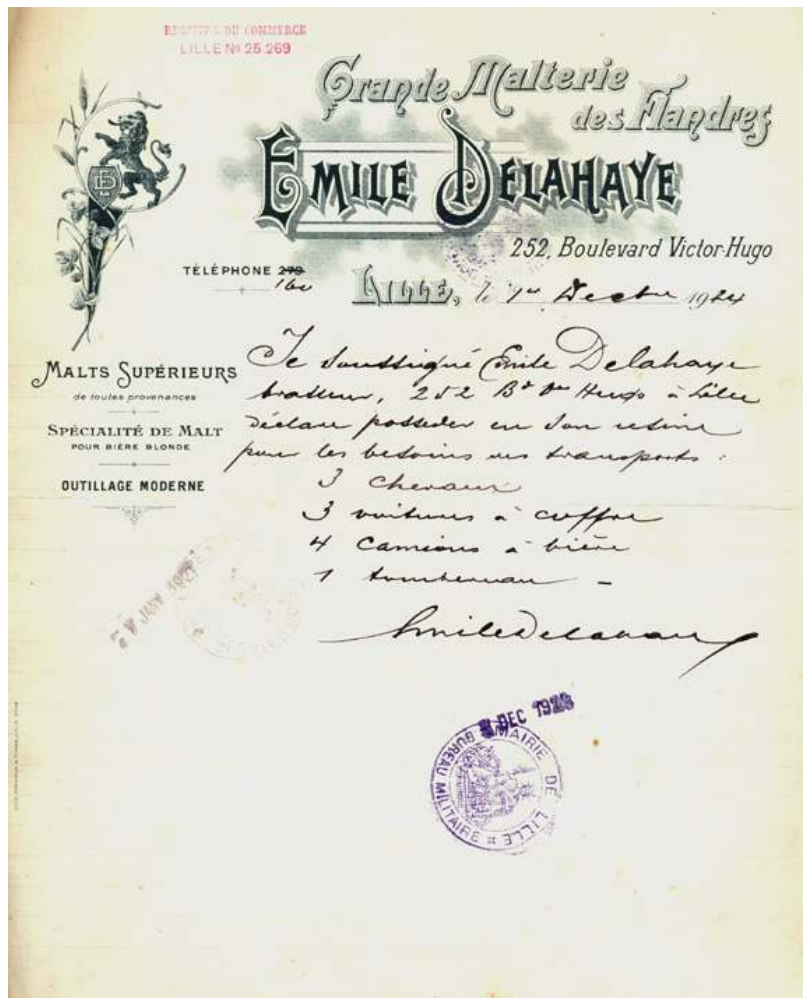

Déclaration de chevaux et voitures par la Brasserie Delahaye à Lille (Nord), 1924.

(c) ANMT, Brasserie Delahaye, 2004034008. 
Figure 10

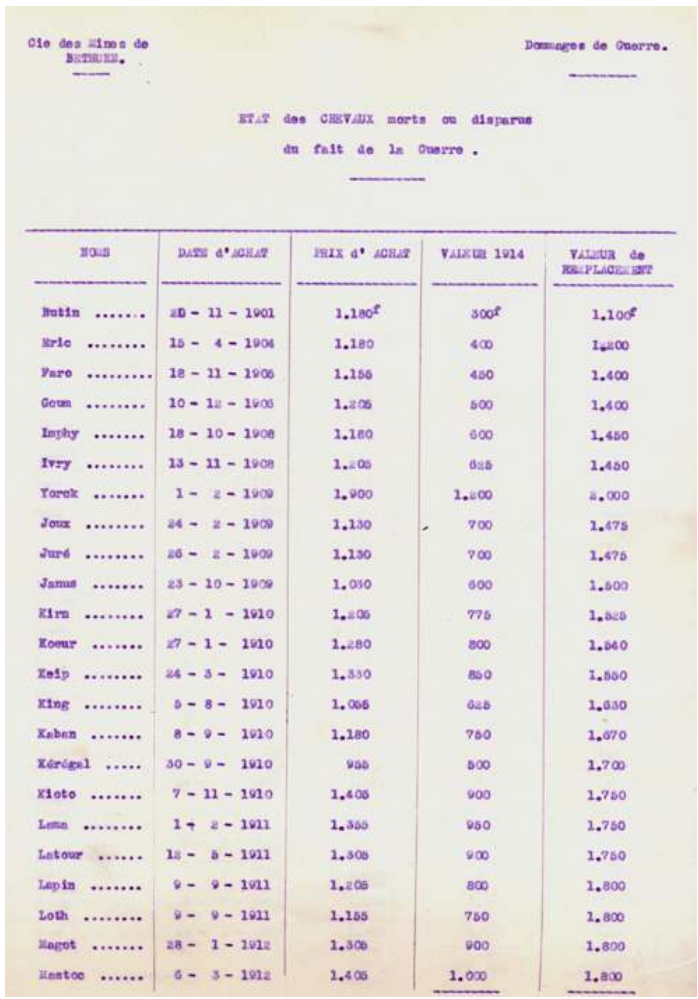

Liste des chevaux de la Compagnie des mines de Béthune morts pendant la Grande Guerre, 1919.

(c) ANMT, Compagnie des mines de Béthune, 1994026608.

En dehors des temps de conflits, le service juridique intervient aussi dans le cadre de la prévention, notamment dans la souscription à des assurances pour la protection des biens de l'entreprise. Ainsi, on découvre de nombreux contrats d'assurance relatifs aux chevaux, à leurs équipements et leurs activités. Ces polices illustrées offrent aussi une représentation intéressante d'équipages de chevaux ce qui est le cas pour celle de Maître Dailly en 1837 qui assure sa malle estafette de Paris au Havre auprès de la Compagnie parisienne d'assurances (fig. $\mathbf{n}^{\circ} \mathbf{1 1}$ ). Ces polices sont une nécessité car il survient de très nombreux accidents de matériels, de personnes et de chevaux. La correspondance reçue par les maîtres des postes Dailly montre que les cochers effectuent des virages trop courts et occasionnent des dommages à d'autres voitures et chevaux et blessent même leur propre attelage. Les chevaux dérapent sur le pavé, se chipent ou se rebellent également. Le récit de ces faits par les victimes donne du relief à ces événements dont on retrouve la trace dans les tableaux des accidents dressés mensuellement ${ }^{9}$ par la Compagnie générale des omnibus. Ils mentionnent systématiquement le numéro d'enregistrement de l'accident, sa date, sa date de déclaration, le numéro de la voiture impliquée, le nom des cochers et des receveurs, la nature, le lieu et le montant des dommages, les sommes admises, le remboursement des cochers. De plus, il ne faut pas oublier que le cheval s'use à la tâche, vieillit et l'entrepreneur doit pouvoir le remplacer sans trop de frais. L'assurer lui permet aussi de ne pas réengager toute la valeur du cheval quelle que soit sa fin. Le système des réformes assurées permet ainsi un roulement moins coûteux des nouvelles recrues. 


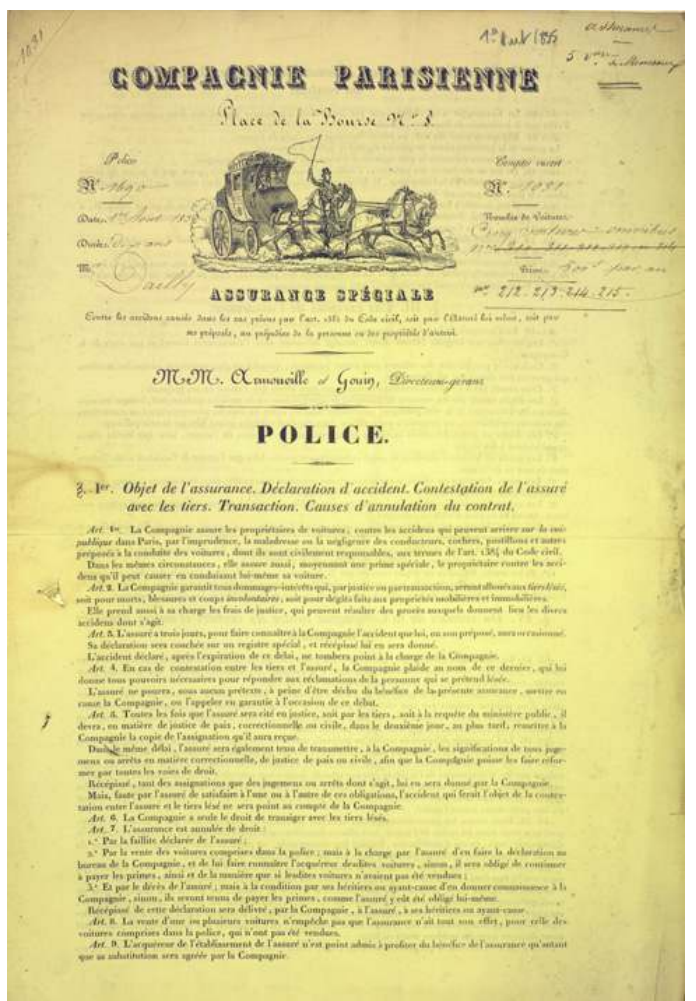

Police d'assurance du maître des postes Dailly souscrite auprès de la Compagnie parisienne, 2 octobre 1837.

(c) ANMT, Maîtres de postes Dailly, 19 AQ 50.

L'écurie, le cheval et son équipement sont aussi sources de nuisances et de pollution en pleine ville. Le service juridique doit alors faire face aux plaignants, souvent le voisinage qui se plaint du bruit et des odeurs liées au fumier entre autres. Plaintes, conflits, compromis se succèdent dans les dossiers générant procès-verbaux de constat, amendes, procès et décisions de justice. L'entreprise doit soit déménager, soit aménager ses locaux. Les plus grandes n'y échappent pas, telle la Compagnie du chemin de fer du Nord qui doit déplacer à plusieurs reprises ses écuries autour de la Gare du Nord à Paris, qui entrent de surcroît en concurrence avec celles de la Compagnie du chemin de fer de l'Est toute proche.

\section{Le cheval : un membre du personnel?}

\section{La constitution des écuries}

En fonction du degré d'attachement de l'entrepreneur à ses chevaux, ces derniers apparaissent ou non dans les archives du personnel. Les entreprises sont très soucieuses de conserver leur nombre de chevaux, voire de l'augmenter en fonction des besoins et des places disponibles dans les locaux. Les achats s'effectuent sur les marchés aux chevaux soit directement soit par intermédiaires, ou bien sur les foires. Le nombre, le sexe (étalon, jument, hongre), l'âge (poulain ou adulte), la race et la couleur sont ainsi contrôlés et minutieusement consignés dans les registres. Il est à noter que ces critères de choix 
dépendent de l'affectation finale du cheval. Les chevaux de trait sont préférés pour les travaux des champs, les chevaux robustes pour les transports en commun et les chevaux plus élégants pour le transport individuel. Pour des missions bien précises, un choix de couleur de robe spécifique et unique peut intervenir comme c'est le cas à la Compagnie générale des omnibus de Paris qui recrute des chevaux de préférence gris (fig. $\mathbf{n}^{\circ} \mathbf{1 2}$ ). Dans le fonds Dizieux et Brosse, maison d'emballage pour l'exportation à Paris, une correspondance suivie de 1904 entre Émile Dizieux et Monsieur Martin, l'intermédiaire pour l'achat des chevaux, montre qu'Émile Dizieux demande l'avis du vétérinaire avant tout achat et précise les critères de son choix (fig. $\mathbf{n}^{\circ}{ }^{13}$ ). Négociation, choix précis et transport par le navire Anatolie via Marseille, peut-être en provenance de Turquie, sont mentionnés, ce qui est rare car les transactions s'effectuaient par oral. Si on conserve des documents relatifs à ces transactions en faible quantité, on ne dispose guère que de chiffres et de statistiques globaux pour les écuries plus importantes, avec plusieurs centaines de chevaux, pour les dépôts des compagnies de chemin de fer (entre 200 et 400) ou d'omnibus ou des postes (plus de dix mille) à Paris. On compte plus de trois millions de chevaux répartis dans le monde agricole.

Figure 12

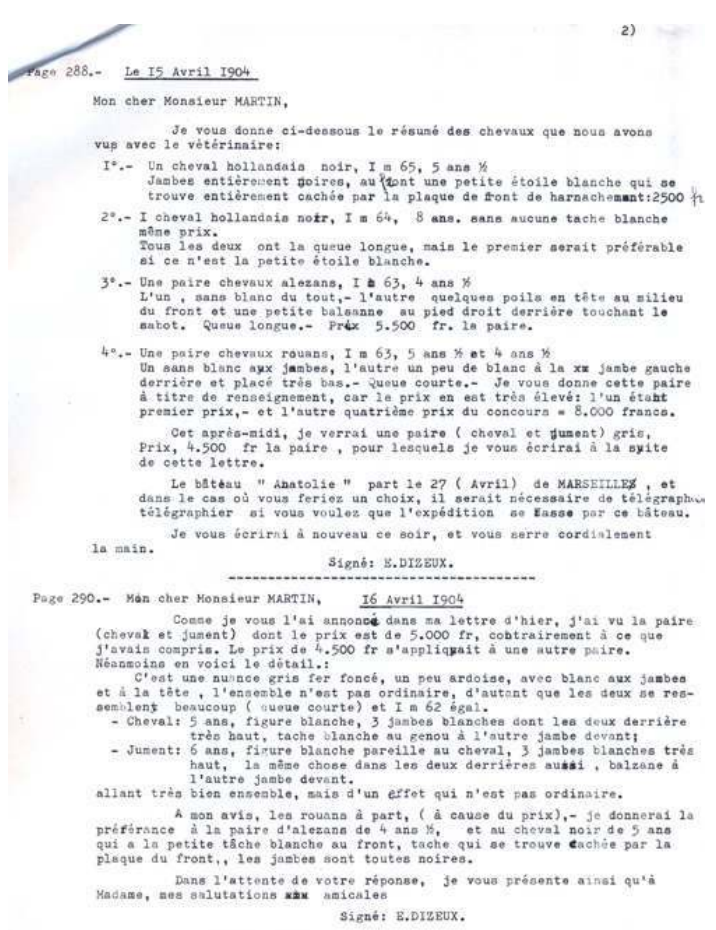

Correspondance relative à l'achat de chevaux pour Dizieux et Brosse, maison d'emballage pour l'exportation à Paris.

(C) ANMT, Dizieux et Brosse, 1996113139 
Figure 13

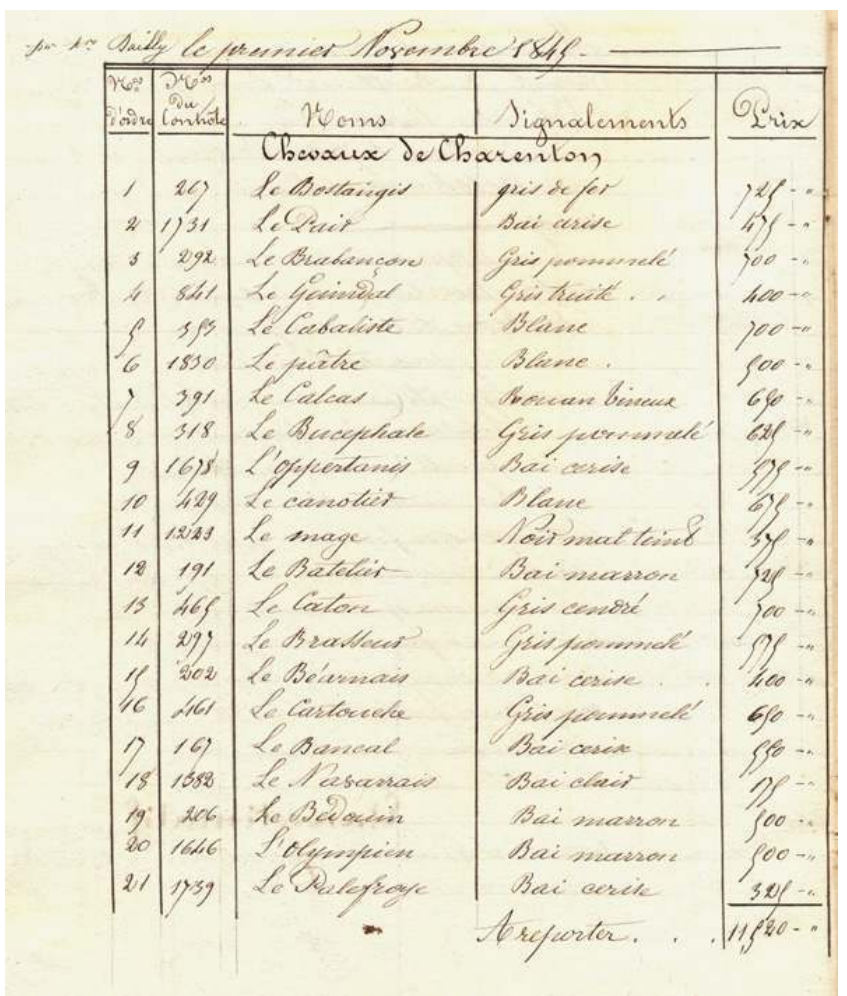

Liste des chevaux de la Compagnie générale des omnibus avec leur nom et leur prix d'achat, 1845. (c) ANMT, Maîtres de postes Dailly, 19 AQ 39.

En dehors de l'achat, les entreprises qui possèdent des fermes peuvent faire saillir leurs juments dans les haras d'État, tels les maîtres de postes Dailly qui élèvent dans leur ferme d'Étuf (Yonne) des juments saillies dans les haras impériaux et leurs poulains, afin de fournir leurs dépôts à Paris (fig. $\mathbf{n}^{\circ} \mathbf{1 4}$ ). La naissance du poulain donne lieu à l'élaboration d'un certificat, véritable état civil avec ascendance et signalement. Les poulains sont ainsi élevés dans les fermes avant de rejoindre leurs aînés dans les activités de l'entreprise. 
Figure 14

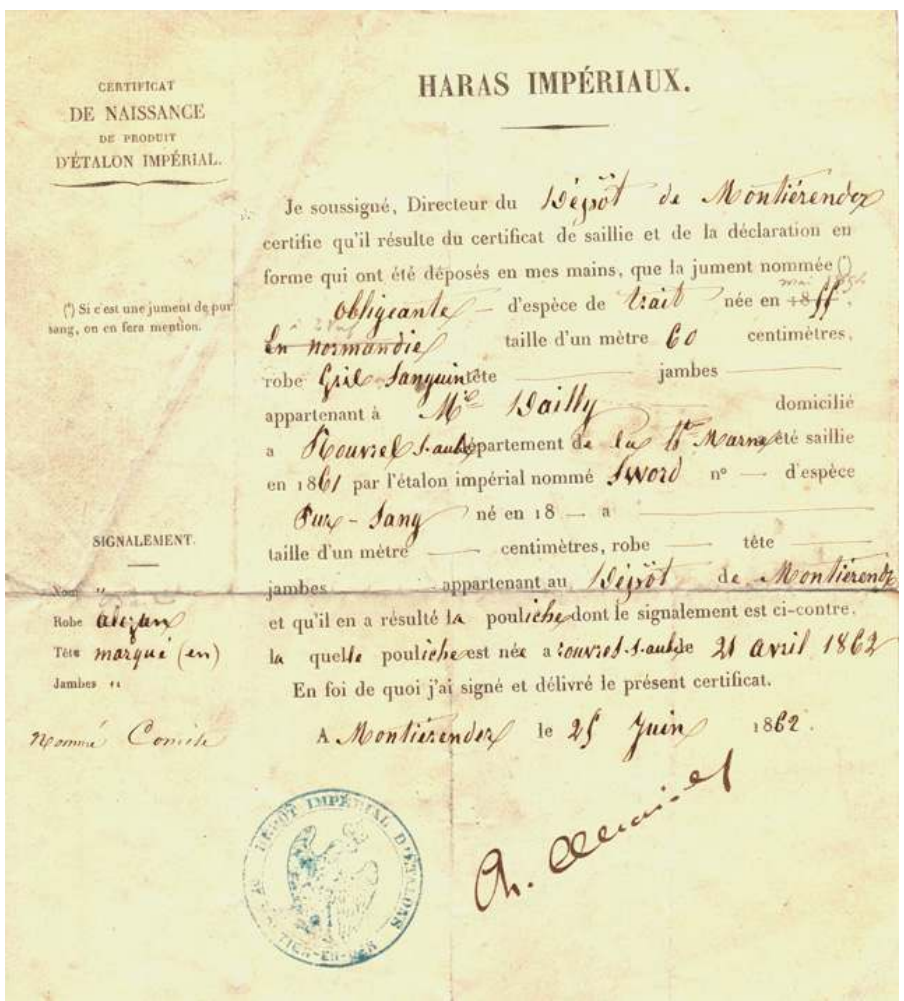

Certificat de naissance d'une pouliche de la Poste aux chevaux de Paris établi par le haras impérial de Montier-en-Der, le 25 juin 1862.

(C) ANMT, Maîtres de postes Dailly, 19 AQ 47.

Les caractéristiques de ces chevaux fraîchement arrivés sont ensuite inscrites dans les registres des écuries : effectifs, matricules, noms, poids, valeurs. Les entrées et les sorties par vente sont mentionnées (fig. $\mathbf{n}^{\circ} \mathbf{1 5}$ ). Les tableaux statistiques hebdomadaires, mensuels et annuels de la Compagnie générale des omnibus détaillent davantage la nature des sorties de chevaux : réformes, restitutions aux marchands, décès dans le délai de garantie, exécutions ou ventes à la boucherie rythment le quotidien des écuries. Ils mentionnent aussi les autres opérations liées à la prise de possession: marquage, raccourcissement de la queue et de la crinière, ferrage. 


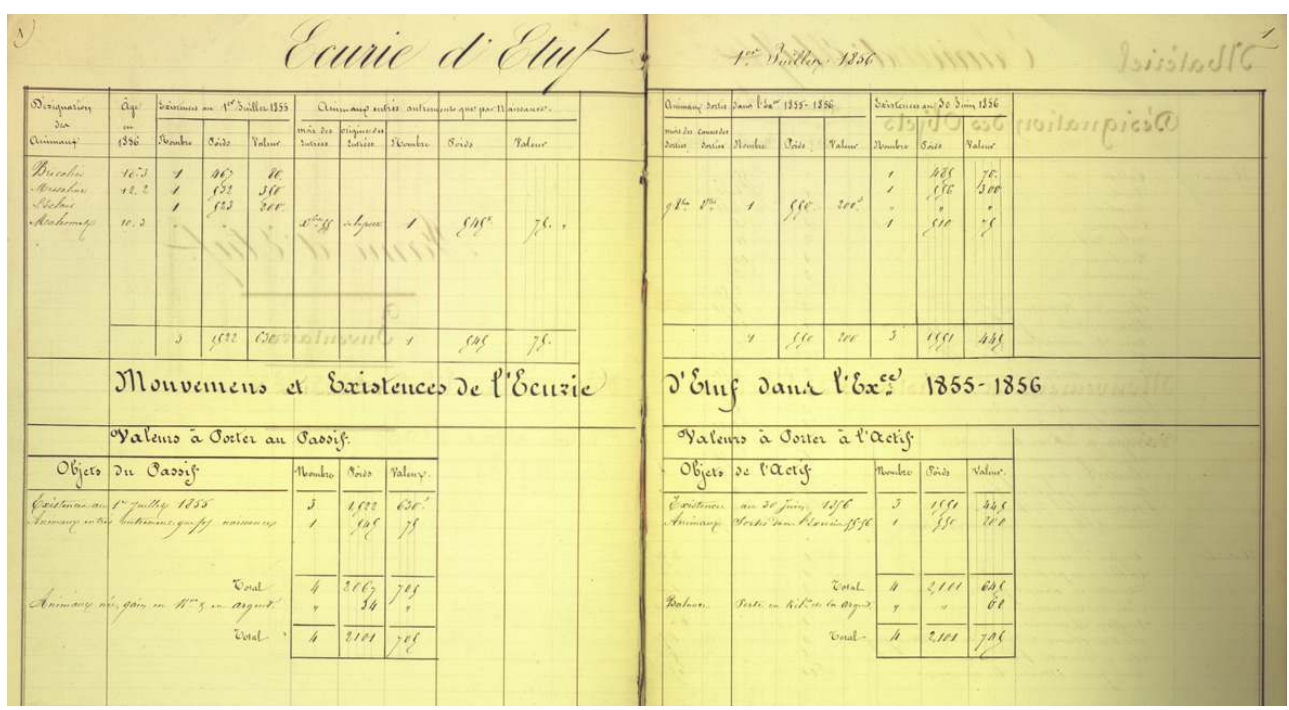

Registre des écuries de la ferme d'Etuf (Yonne), appartenant aux maîtres des postes Dailly, 1856. (c) ANMT, Maîtres de postes Dailly, 19 AQ 47.

\section{Les métiers du cheval : des personnels spécifiques}

Les archives des entreprises conservées aux ANMT donnent quelques éléments sur les intermédiaires entre le lieu de production, la vente et les lieux d'achat et d'activité du cheval. La correspondance, les tableaux de remonte, les factures, les registres de comptes renseignent sur les filières, les lieux géographiques et surtout sur les métiers du cheval. On retrouve des éleveurs, des marchands équins et des convoyeurs de chevaux qui agissent au sein de leur société ou sur des marchés à chevaux. Une fois le cheval arrivé à destination, d'autres professions interviennent et ce directement dans l'intimité du cheval: l'éleveur, le fermier, le palefrenier, le vétérinaire. Le registre du personnel énumère ces acteurs avec leur nom, la date de leur entrée et de leur sortie (fig. $\left.\mathbf{n}^{\circ} \mathbf{1 6}\right)$. Des notes sur le service vétérinaire au sein de la Compagnie des mines de Béthune décrivent son organisation, le rôle du vétérinaire et de son aide. La correspondance révèle le nom de ces derniers et leurs activités quotidiennes avec les difficultés qu'ils rencontrent par rapport aux notes d'organisations théoriques (fig. $\mathbf{n}^{\mathbf{0}} \mathbf{1 7}$ ). 
Figure 16

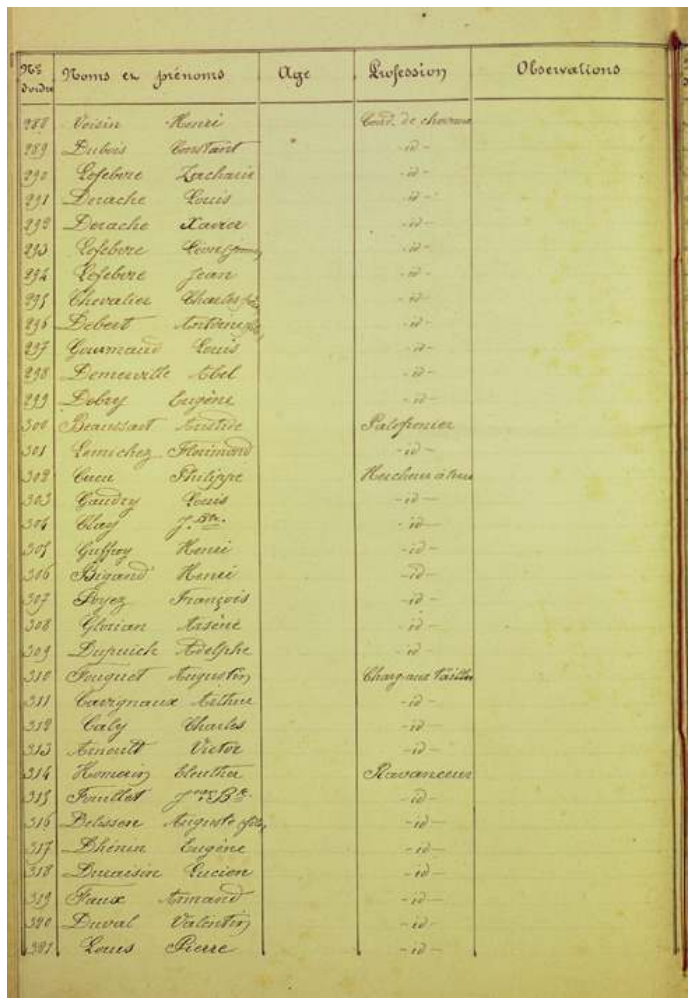

Extrait du registre du personnel du jour et du fond des Mines de Béthune, 28 mars 1885. (c) ANMT, Compagnie des mines de Béthune, 1994026291. 
Figure 17

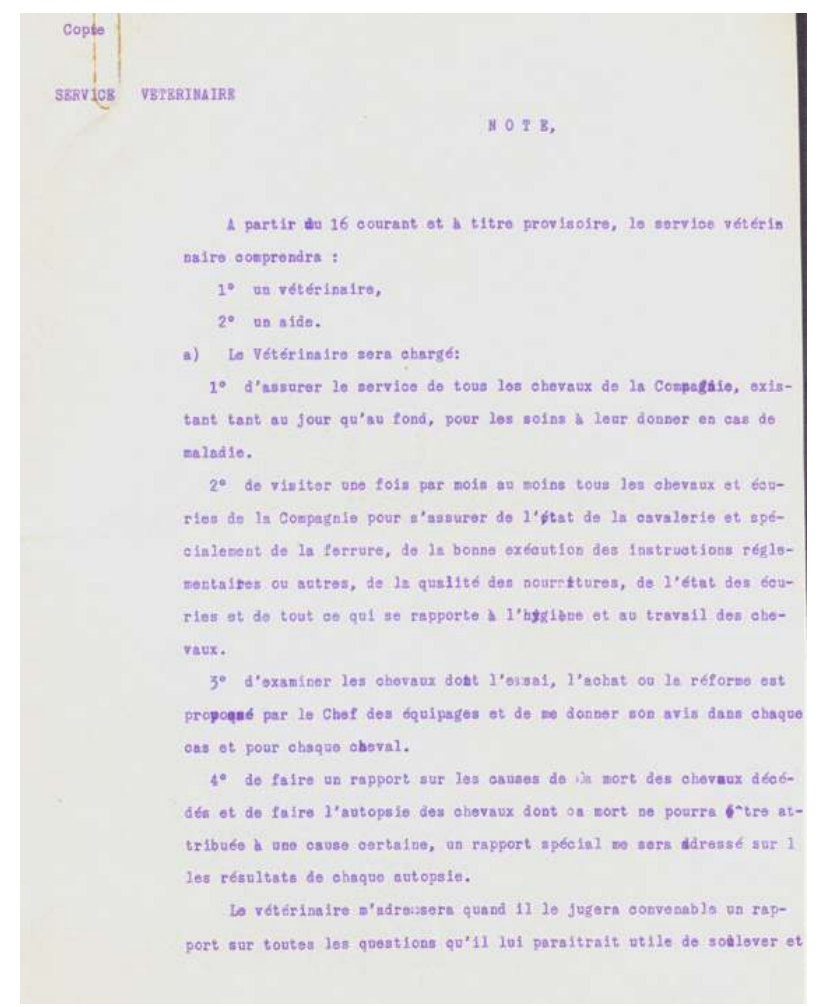

Note d'organisation du service vétérinaire, 15 mars 1904.

(c) ANMT, Compagnie des mines de Béthune, 1994026300.

De plus, les métiers qui permettent de fabriquer l'équipement du cheval apparaissent au détour des registres des magasins de maréchalerie (fig. $\mathbf{n}^{\circ} \mathbf{1 8}$ ), des listes de fournitures pour le forgeron et des listes des matériaux utilisés (cuir, essences de bois, quincaillerie, etc.) par le charron (fig. $\mathbf{n}^{\circ} \mathbf{1 9}$ ). Le sellier apparaît dans les catalogues de vente et des inventaires d'achat de brides, selles, mors, sangles, étriers, etc. Ces professionnels peuvent être géographiquement proches des entreprises si ces dernières sont suffisamment importantes, comme les compagnies minières ou les compagnies de chemin de fer, ou plus ou moins éloignés pour les petites entreprises qui ont recours à des prestataires externes. 


\section{Figure 18}

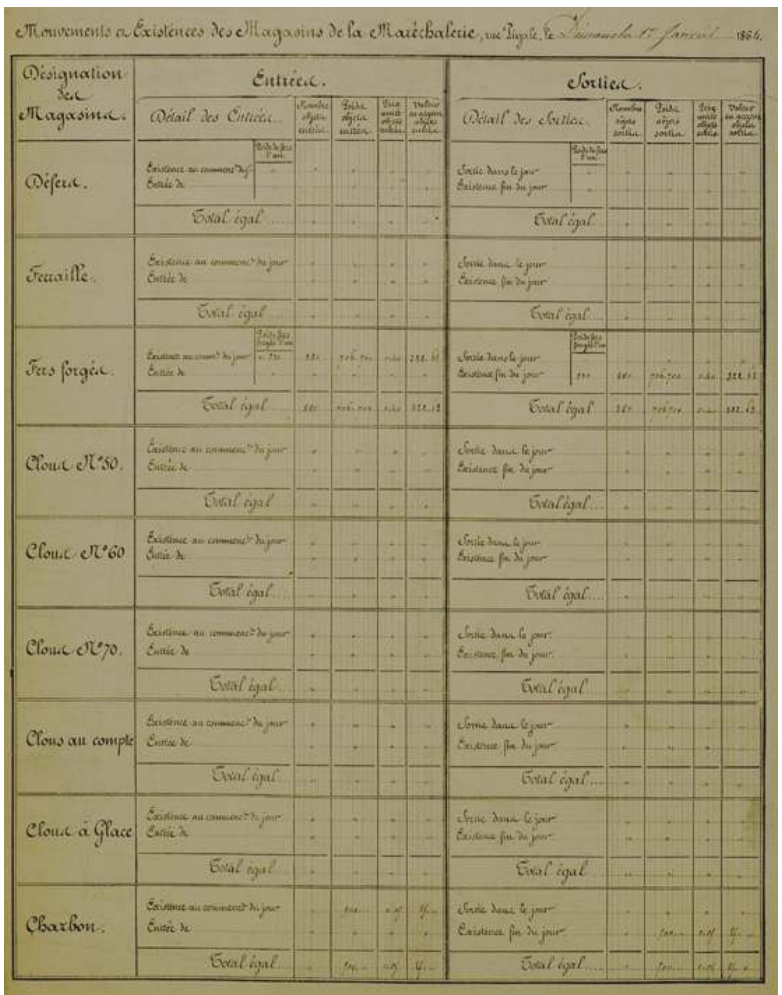

Registre des magasins de la maréchalerie de la Poste aux chevaux à Paris, 1864. (c) ANMT, Maîtres de postes Dailly, 19 AQ 36. 
Figure 19

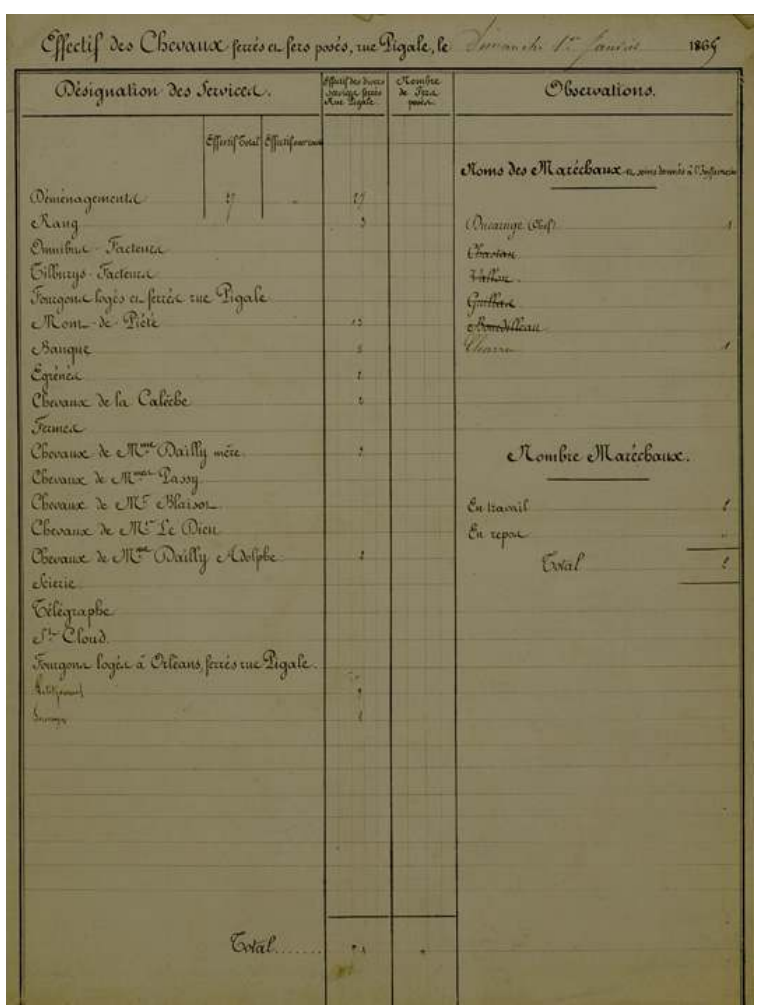

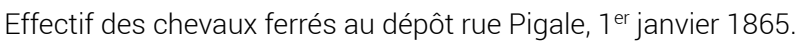

(c) ANMT, Maîtres de postes Dailly, 19 AQ 36.

Enfin, les archives recèlent des informations sur les professions qui font travailler le cheval. En premier lieu, l'agriculteur qui utilise le cheval pour le labour, la récolte, le pressage des fruits et le transport, comme le montre le rapport du régisseur des cultures de la Colonie agricole de Saint-Maurice en $1887^{10}$. Dans les mines, le conducteur de cheval est le collègue de travail du cheval qui tracte en surface comme au fond les wagonnets remplis de charbon. Sa spécificité est mentionnée dans le registre du personnel de la mine. Elle est bien distincte du palefrenier. Chaque mine compte une dizaine de conducteurs de chevaux voire davantage en fonction de la taille de l'exploitation.

En ville, ce sont les charretiers, les cochers et les postillons qui conduisent les chevaux. Le livre des gages des cochers de la Poste aux chevaux de Paris renferme mensuellement l'émargement, le nom, le temps de travail, le prix de la journée et la paie à donner. De même pour les facteurs qui, à travers le registre des tournées, laissent leur identité, leur temps de travail et le nombre de chevaux utilisés. Le livret de postillon livre des informations sur celui qui conduit, tracte la voiture avec le courrier et les passagers et qui entretient les chevaux, précise l'état-civil et le parcours professionnel de l'agent. Certains sont novices, d'autres, comme Jean Vapard (fig. $\mathbf{n}^{\circ} \mathbf{2 0}$ ) (fig. $\mathbf{n}^{\circ} \mathbf{2 1}$ ), ont une longue expérience. 


\section{Figure 20}

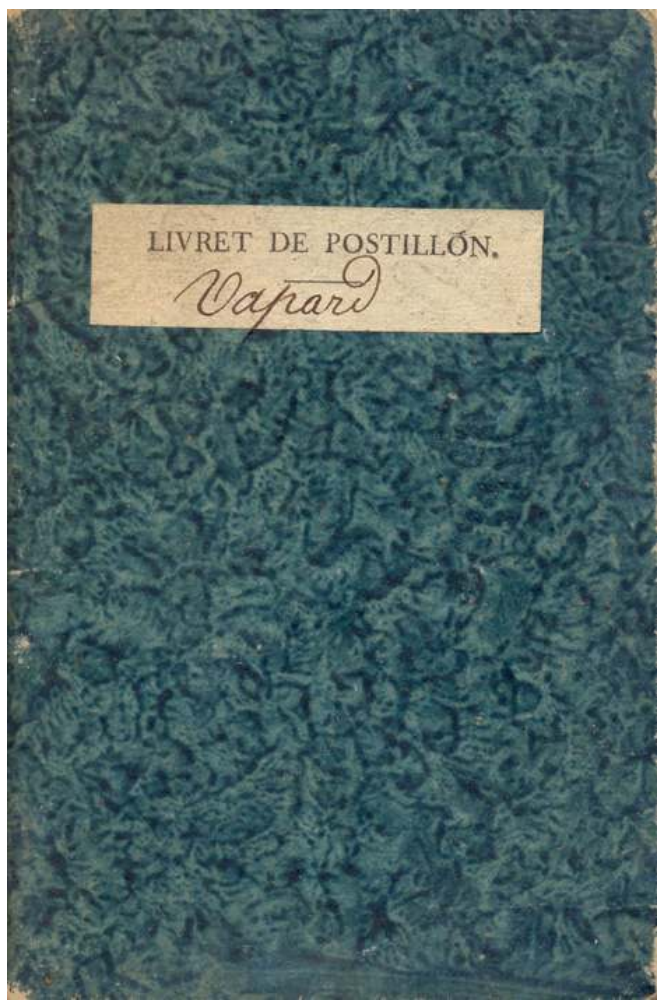

Livret du postillon Jean Vapard de la Poste aux chevaux de Paris, 1822.

(c) ANMT, Maîtres de postes Dailly, 19 AQ 37. 


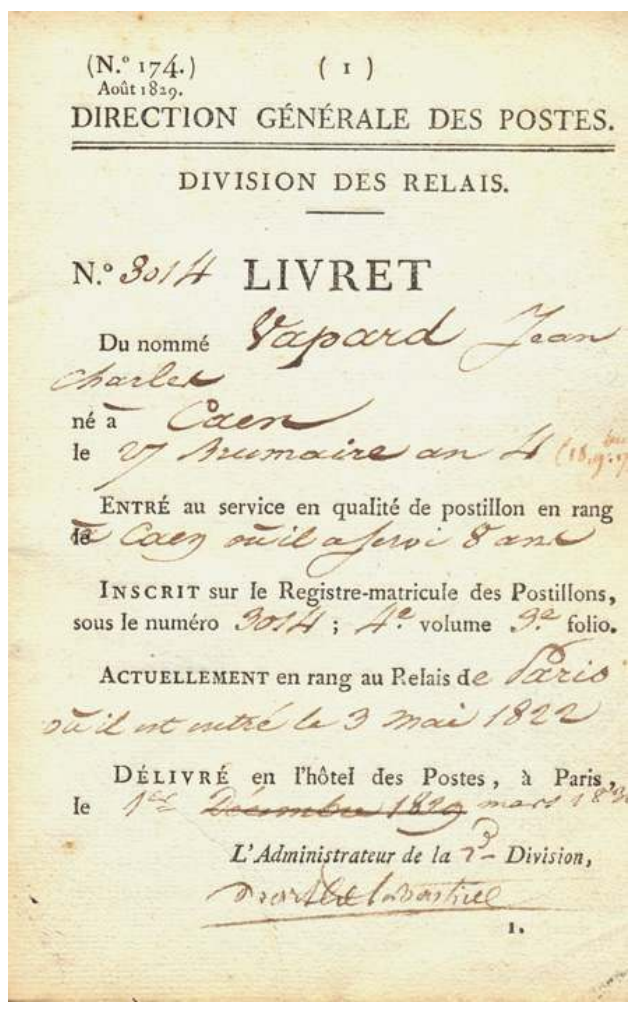

Livret du postillon Jean Vapard de la Poste aux chevaux de Paris, 1822.

(c) ANMT, Maîtres de postes Dailly, 19 AQ 37.

Tous ces professionnels, quelle que soit leur proximité avec le cheval, sont les acteurs indispensables pour préparer, entretenir, soigner et faire travailler ce dernier.

\section{Le cheval au quotidien : une force motrice aux emplois multiples}

\section{Les hippoactivités}

$\mathrm{Au} \mathrm{XIX}^{\mathrm{e}}$ siècle et dans la première moitié du $\mathrm{XX}^{\mathrm{e}}$ siècle, le cheval est présent partout au travail, à la campagne comme à la ville, ce dont témoignent les représentations iconographiques contenues dans les fonds des ANMT mais aussi les registres de production des entreprises qui les emploient. Il se retrouve en France, en Europe, jusqu'au Maghreb, en Asie et en Amérique latine. Ailleurs, comme en Afrique, les entreprises ont recours à la force humaine exploitée en grand nombre et à peu de frais. Le cheval fait partie des animaux de travail, au même titre que certains bovidés, et se distingue des animaux de rente, produisant lait, œufs, viande, peaux, laine ou engrais. Il constitue la force motrice musculaire indispensable pour réaliser les activités agricoles et industrielles.

Avant d'être utilisé dans les activités humaines, il doit être conditionné à ses futures missions et ce dès le plus jeune âge. C'est pourquoi l'agriculteur ou le fermier habitue le poulain au port du harnais, de la bride, à la traction et au commandement à la voix. Dans les mines, le cheval commence par être acclimaté au bruit, aux odeurs, à la poussière, au 
contact avec l'homme et avec les wagonnets à tirer. Une fois dressé, il peut être descendu au fond (fig. $\mathbf{n}^{\circ} \mathbf{2 2}$ ). La Compagnie générale des omnibus de Paris fait même appel à une école de domptage pour ses chevaux qui se retrouvent en pleine circulation.

Figure 22

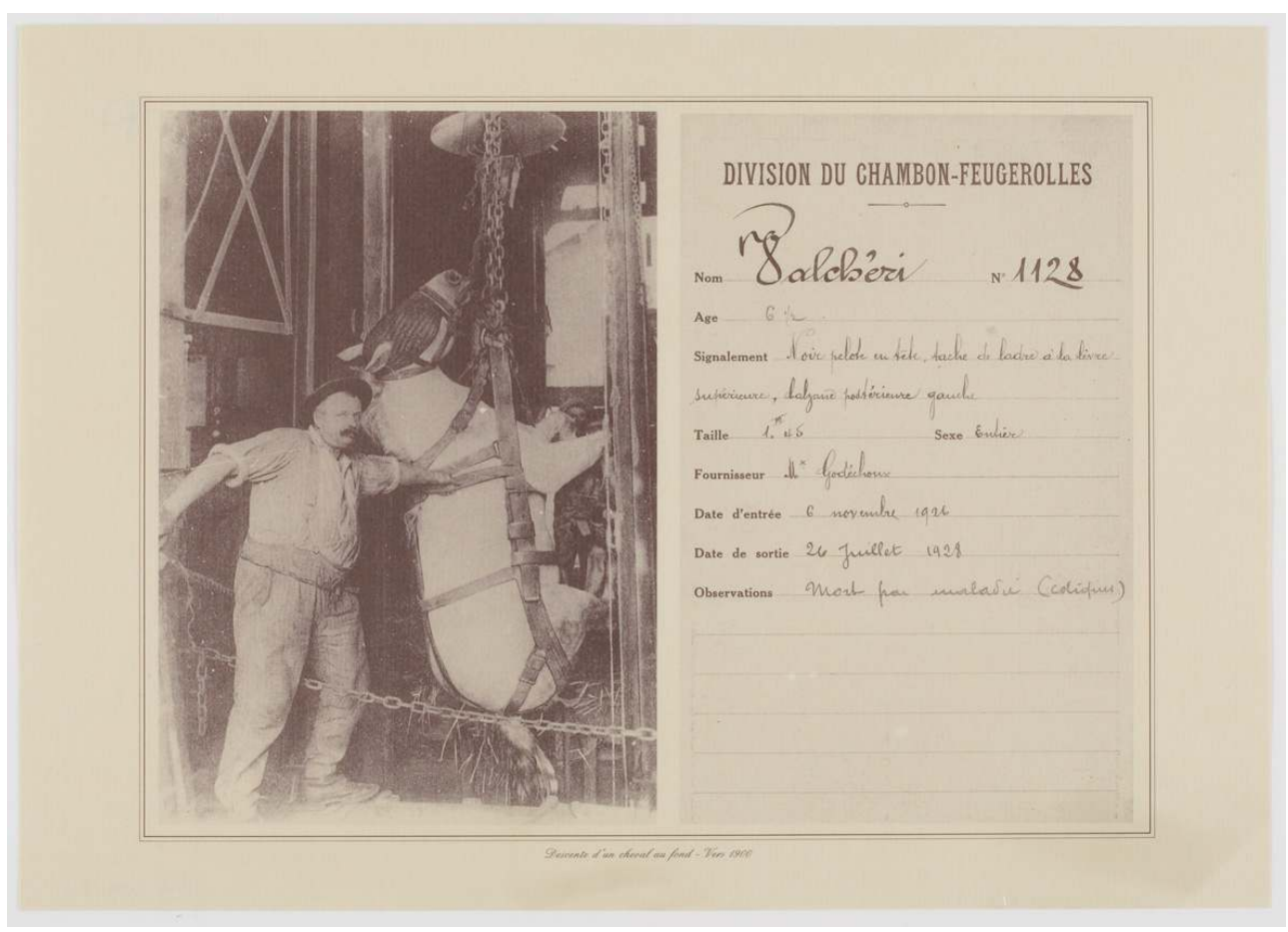

Houillères du bassin du Centre et du Midi, division de Chambon-Feugerolles, descente d'un cheval au fond de la mine, vers 1900.

(c) ANMT, Fonds Jacques Petitmengin, 1998019051.

31 Ainsi préparé, il peut ensuite remplir trois fonctions : tirer, transporter et actionner. La traction dans le monde agricole de charrue, herse, etc., est indispensable aux travaux des champs, labours et moissons entre autres (fig. $\mathbf{n}^{\circ} \mathbf{2 3}$ ). L'hippotraction vient également aider les bateaux dans les cours d'eau, les canaux, pour leur guidage et leur remorquage. 


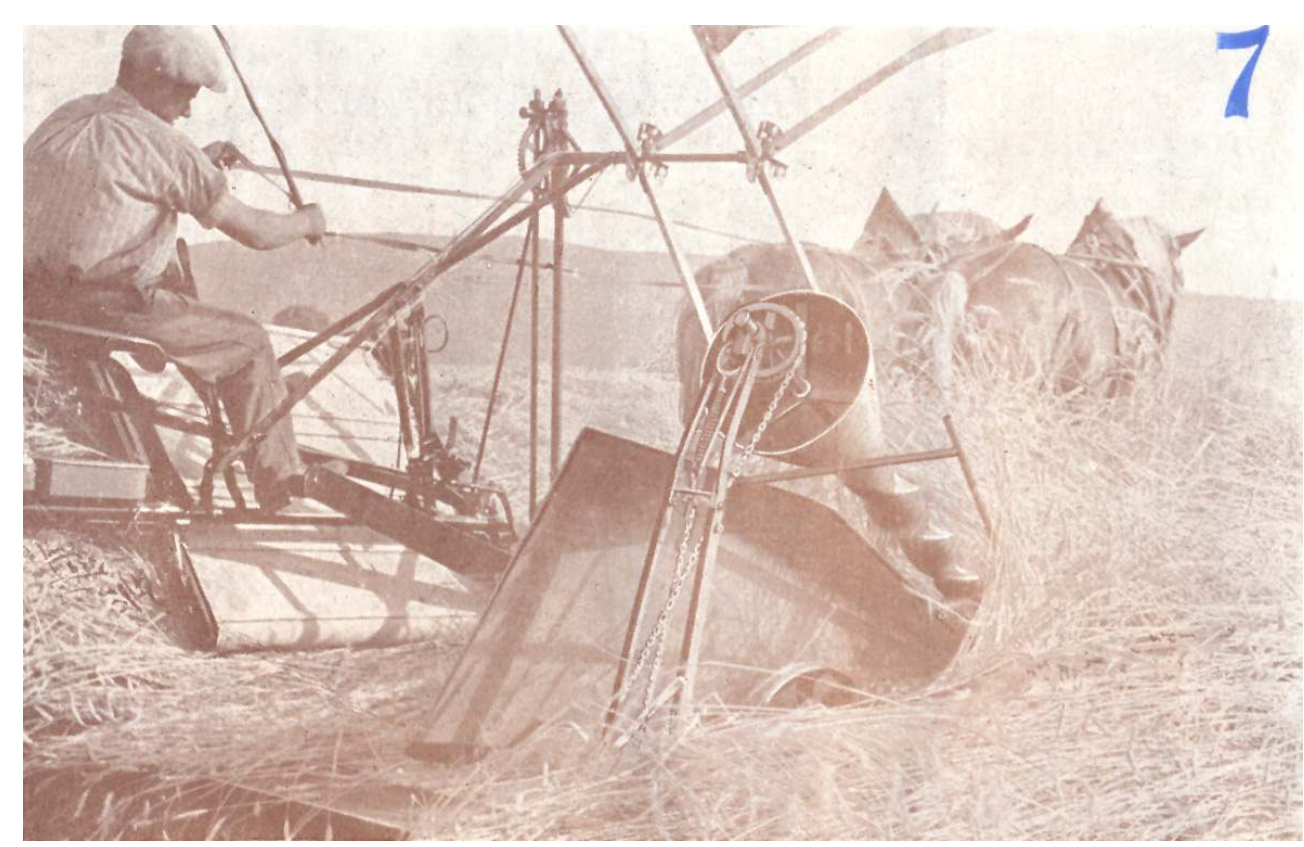

Moissonneuse Savary, années 1950.

(c) ANMT, Établissements Savary, 2009029023.

Le cheval est utilisé pour le transport des matières premières, des marchandises et des voyageurs avec toutes sortes de voitures nécessitant un attelage comprenant un ou plusieurs chevaux. Le cheval est présent dans les champs pour transporter les récoltes; dans les mines et les carrières pour les matériaux d'extraction (charbon, minerai, pierre, sable, etc.) ; dans l'industrie pour les matières premières et produits transformés (agroalimentaire, textile, sidérurgie, etc.) ; dans le commerce (marchés, commerçants, etc.) ; dans la construction (approvisionnement en matériaux, déblaiement de gravats, etc.) et dans les transports (routiers individuels ou collectifs, en appoint ou en complément des chemins de fer) (fig. $\mathbf{n}^{\circ} \mathbf{2 4}$ ). Le cheval, seul ou en équipage, est ainsi présent partout où l'homme ne peut intervenir et partout où la machine motorisée appropriée n'est pas efficace ou n'existe pas encore, et ce pour des raisons d'accès, d'agilité et de précautions vis-à-vis de la marchandise ou de la tâche à accomplir. Le cheval complète les machines et les activités humaines. Dans le transport routier, son emploi de plus en plus organisé dans les villes dans les transports en commun oblige bientôt les autorités à réglementer la circulation entre les voitures et les chevaux, face aux automobiles et aux tramways qui font leur apparition à la fin du XIX ${ }^{\mathrm{e}}$ siècle. Le dernier omnibus à cheval circule en janvier 1913 à Paris. Il s'efface derrière les tramways électriques et le métro. 


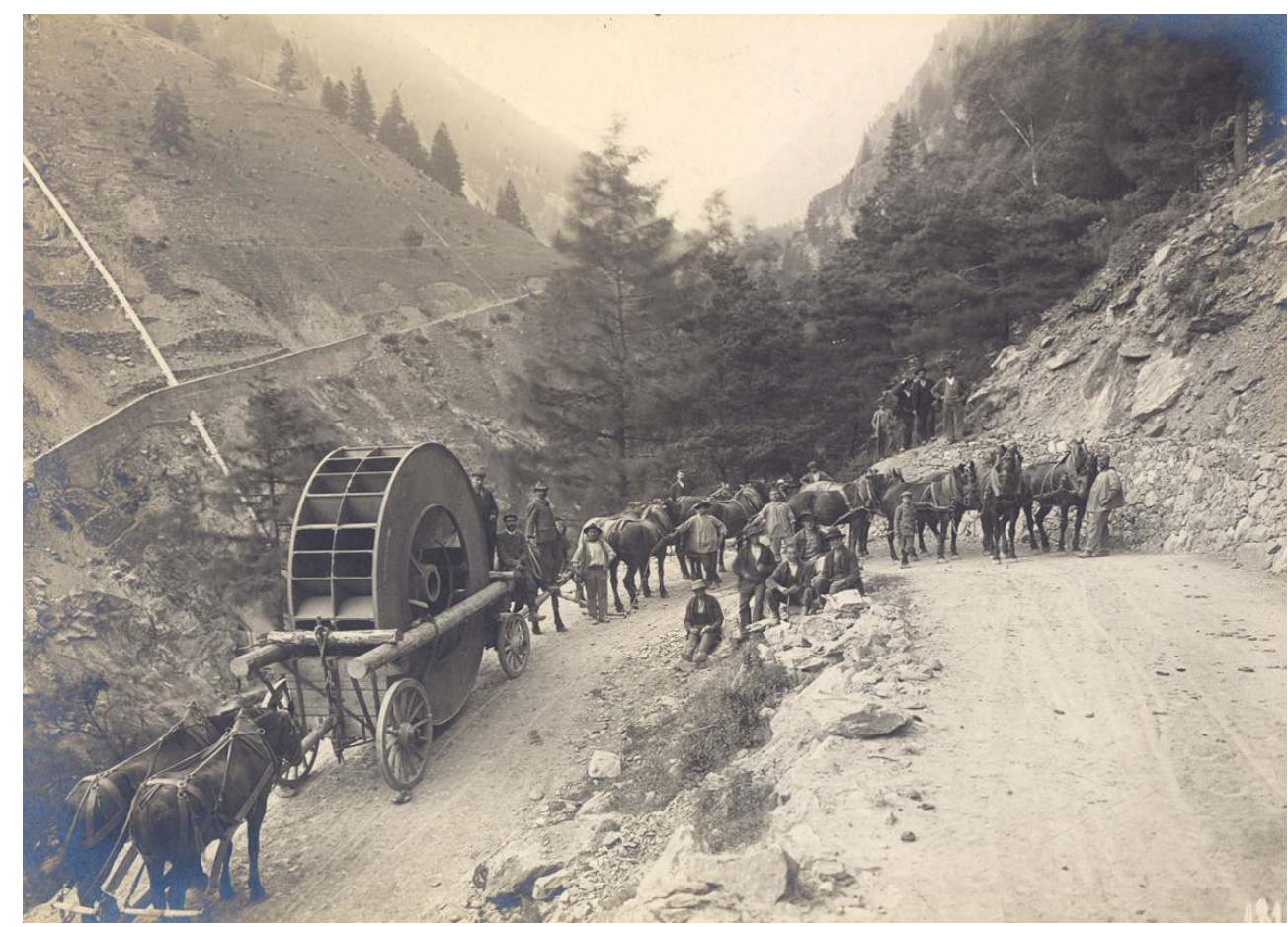

Transport des aubes des ventilateurs pour le tunnel du Simplon sur la ligne de chemin de fer BerneSimplon, 1907.

(c) ANMT, Chagnaud Construction, 2007009006.

33 L'actionnement de machines (meules, moulins, batteuses, rouages de pressoir ou de brasserie, machines hydrauliques, etc.) est la seconde mission du cheval. Il est ainsi présent sur les chantiers de construction mais au fur et à mesure que se généralisent les engins de levage, les grues et autres machines, - le cheval se retrouve relégué en périphérie des chantiers, aux tâches de transport.

34 Les fonds des ANMT évoquent aussi de nombreux exemples d'attelages. Ces chevaux sont de races plus fines et sont particulièrement soignés (tonte, tresses, harnachement luxueux, couvertures, etc.) (fig. $\mathbf{n}^{\circ} \mathbf{2 5}$ ). 


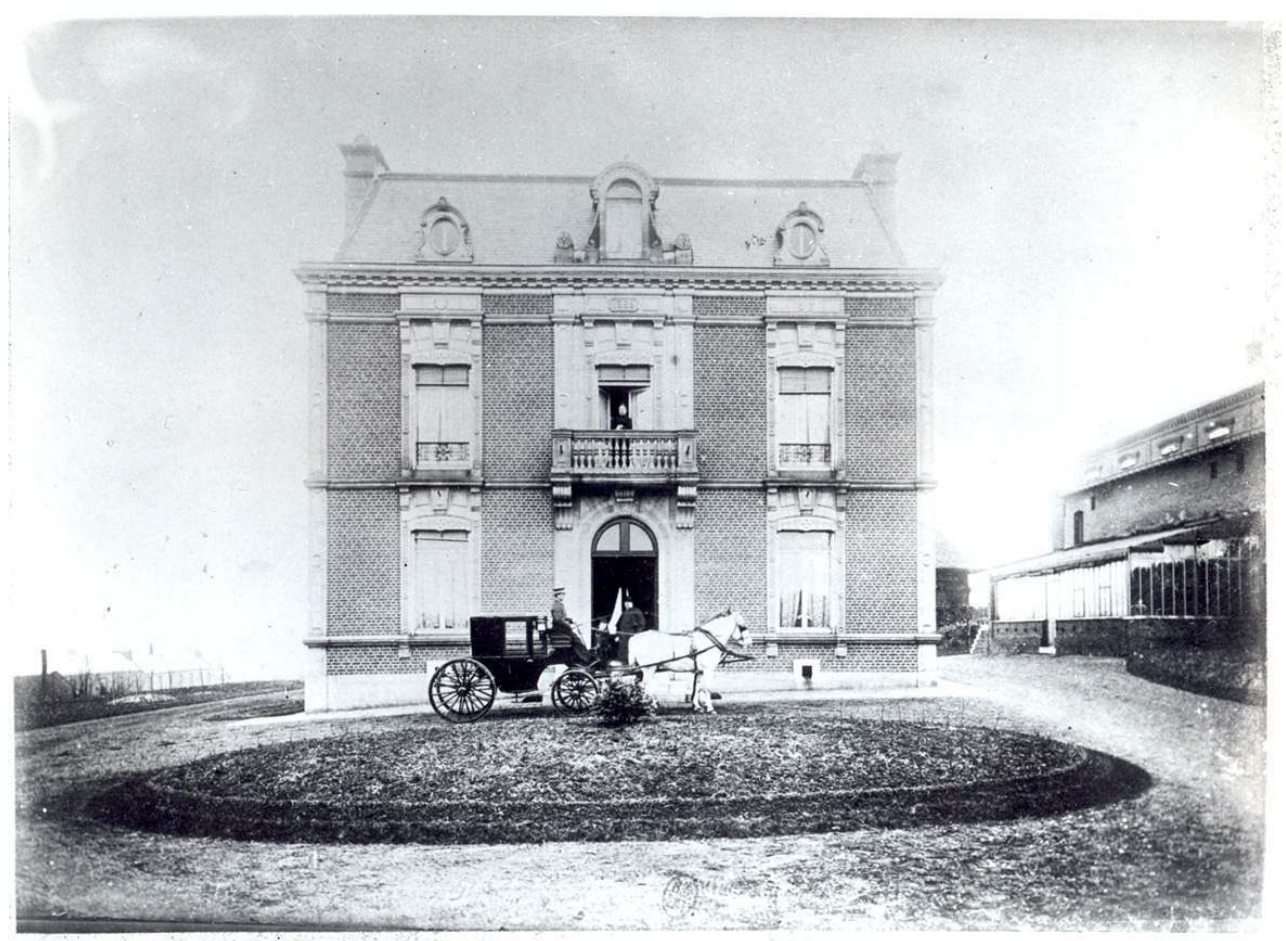

Calèche du directeur des Mines de Bruay attendant devant sa maison, 1889.

(c) ANMT, Charbonnages de France, 200700814446.

Le travail des chevaux est rapidement chiffré, évalué dans des notes et des registres (fig. $\mathbf{n}^{\circ} 26$ ). Des traités, notamment pour l'exploitation des mines ${ }^{11}$, théorisent l'utilisation optimale et efficace des chevaux. Le rendement est calculé en rapport entre tonnage, temps de parcours réel, et en distance parcourue. Des unités de mesure apparaissent: cheval indiqué, cheval effectif ou réel, cheval nominal ou cheval vitesse, etc. 


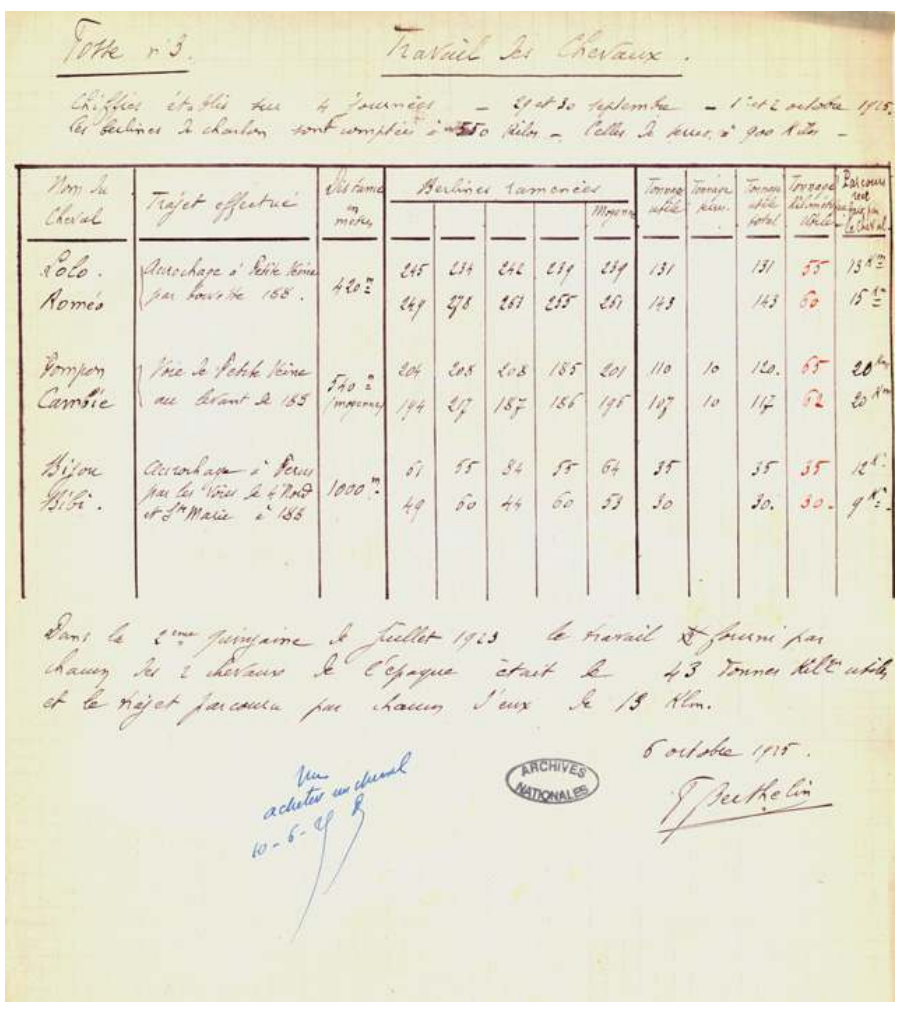

Note sur le travail des chevaux aux Mines d'Ostricourt et Carvin, 1925.

(C) ANMT, Compagnie des mines d'Ostricourt et de Carvin, 1994057054.

On note par ailleurs une sorte de hiérarchisation entre les chevaux: cheval agricole, cheval moteur dans l'industrie, cheval de transport de marchandises ou collectif, cheval de transport de calèches. Les races, le sexe, la couleur de la robe et l'équipement les distinguent. Cependant, ils sont tous aussi utiles et précieux aux yeux de leur propriétaire, ce qui nécessite attention et traitement particulier par rapport à l'outillage.

\section{Entretien et soins}

Le cheval, outil onéreux et précieux, est néanmoins fragile. Il requiert un soin particulier car il est confronté à un environnement très salissant, fatigant et dangereux. Le premier soin est l'alimentation. Le registre des écuries des fermes des maîtres de postes Dailly consigne scrupuleusement le nombre de rations, leur quantité et leur nature : avoine, foin, luzerne. D'autres aliments sont introduits pour les chevaux des mines afin d'enrayer les effets néfastes de la poussière de charbon mortelle à la longue : carottes fourragères et potagères, navets, céleris rave, lentilles sont utilisés comme dépuratifs (fig. $\left.\mathbf{n}^{\circ} \mathbf{2 7}\right)\left(\mathbf{f i g} \mathbf{~} \mathbf{0}^{\circ}\right.$ 28). Ces aliments proviennent de leurs propres exploitations qui servent de laboratoires d'essai. La Compagnie générale des omnibus de Paris se lance aussi dans des expériences d'alimentation parfois artificielles : broyage, hachage, fermentation, cuisson. Par ailleurs, ces entreprises sont démarchées par des marchands d'alimentation pour chevaux à base de mélasse et de sucre, comme Le Pail'mel qui entreprend en 1907 les maitres des postes Dailly en vain. Les essais et les théories d'alimentation équine se retrouvent dans les périodiques agricoles, tel le Journal de l'agriculture, largement diffusés et conservés dans le 
fonds des maîtres des postes Dailly car faisant partie de leur bibliothèque. Les sources présentes aux ANMT sont très discrètes concernant le problème de l'eau car il faut environ 30 litres d'eau par jour et par cheval. On le devine toutefois à travers les plans des écuries, les adductions et les fontaines.

Figure 27

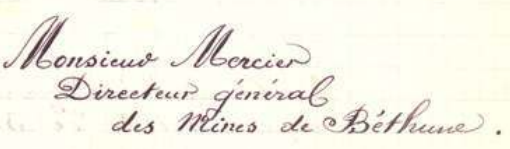

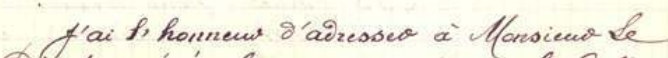
Derecterio g'enéral, un rapport on la Cecltare de la Comprocginic, concennangs la récolte de 1899

Les terrains cultives par la Conpragnice, en $18 \mathrm{gg}$, comprenainy 61..01 are.

bes soixante of in heetares oe trouvaina ziprastis sur les tersitoires de Bully, de Guenay, de Loos et de Mazuigarbe', de la manivie scivande:

$19^{\text {hut }} 84^{\circ}$. $80^{\circ}$ sue Bully.

$7^{h} 53^{\circ}$. $50^{\circ}$ sur Grovery soos

33 ol: to sur Mazeingarbe.

Rapport d'exploitation des fermes de la Compagnie des mines de Béthune, 1899.

(c) ANMT, Compagnie des mines de Béthune, 1994026599. 


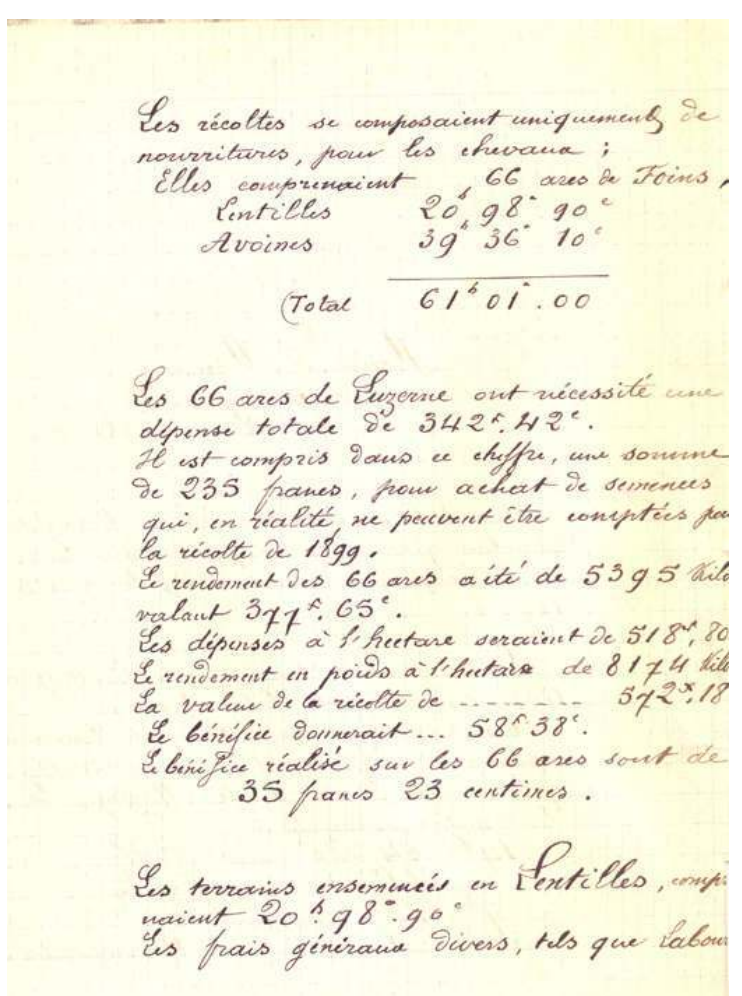

Rapport d'exploitation des fermes de la Compagnie des mines de Béthune, 1899

(c) ANMT, Compagnie des mines de Béthune, 1994026599.

Le deuxième soin est la confection d'une litière propre et confortable dans leur stalle ou box à base de paille qu'il faut nettoyer régulièrement et changer. À la campagne, le fumier est transformé en engrais dans la ferme, il est scrupuleusement compté et réparti au même titre que celui produit par les autres animaux. À la ville, il est évacué rapidement pour évite les nuisances, soit vers ces exploitations soit par des spécialistes rémunérés pour cette tâche (fig. $\mathbf{n}^{\circ} \mathbf{2 9}$ ). L'espace public, urbain ou non, intègre le cheval différemment au XIX siècle. La réglementation, en plus de garantir une meilleure circulation, cherche à assainir cet espace en tentant de mieux contrôler cette vie animale. 


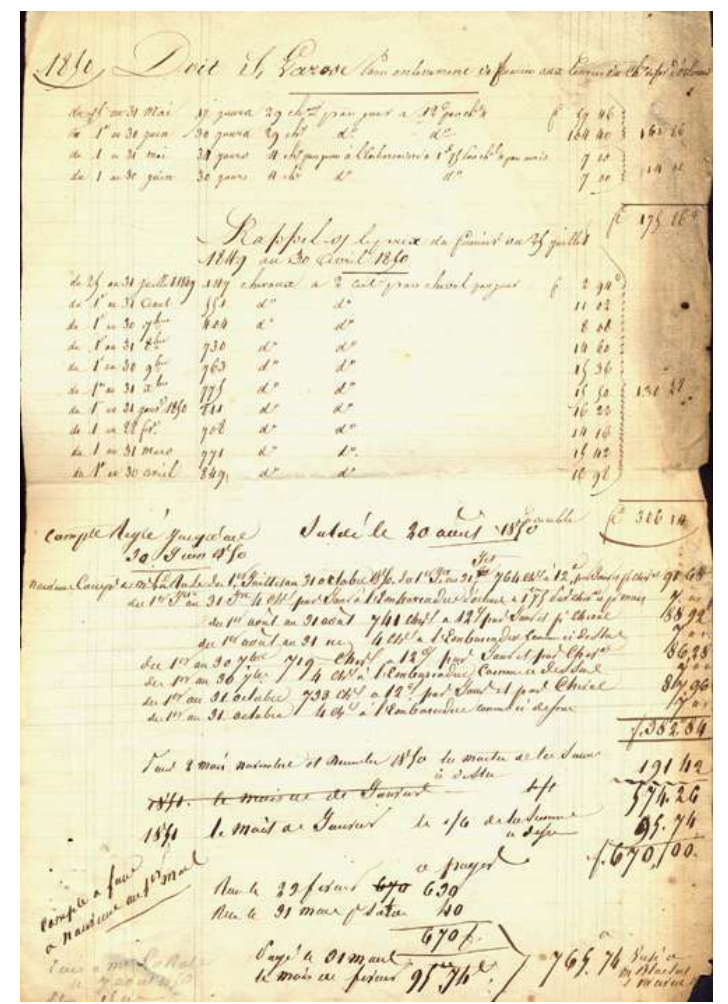

Facture adressée à la société S. Larose pour l'enlèvement du fumier des écuries Dailly dans les locaux du Chemin de fer du Nord à Paris, 1850.

(C) ANMT, Maîtres de postes Dailly, 19 AQ 35.

Le troisième soin consiste en des gestes d'hygiène corporelle au retour du travail: brossage, bain, massage (fig. $\mathbf{n}^{\circ} \mathbf{3 0}$ ). Il faut prévenir les blessures par frottement entre harnachement et peau qui peuvent être favorisées par la présence de poussière abrasive. Les séances de soin quotidien sont aussi l'occasion de détecter des pathologies (troubles digestifs, infections, etc.), les infestations par des parasites (teigne, gale, vers, etc.) et surtout les signes de fatigue intense, les blessures, les déformations et les handicaps (cécité, jambes arquées, etc.). Ces différentes affectations nécessitent l'intervention du vétérinaire. Les grandes entreprises font appel à leur propre vétérinaire comme la Compagnie des mines de Béthune qui est équipée d'une salle d'opération munie d'une table-bascule pour le cheval. Sinon, un vétérinaire extérieur est appelé au cas par cas. Le vétérinaire, quel que soit son statut, peut opérer, apposer des pansements, prescrire un régime alimentaire particulier, des médicaments, voire une cure. À cet effet, la Compagnie générale des omnibus de Paris dispose d'une ferme hôpital à Claye-Souilly d'une capacité de 300 à 600 chevaux. Le vétérinaire, comme le soigneur, peut décider de la réforme, de la vente ou de l'abattage via la boucherie. 
Figure 30

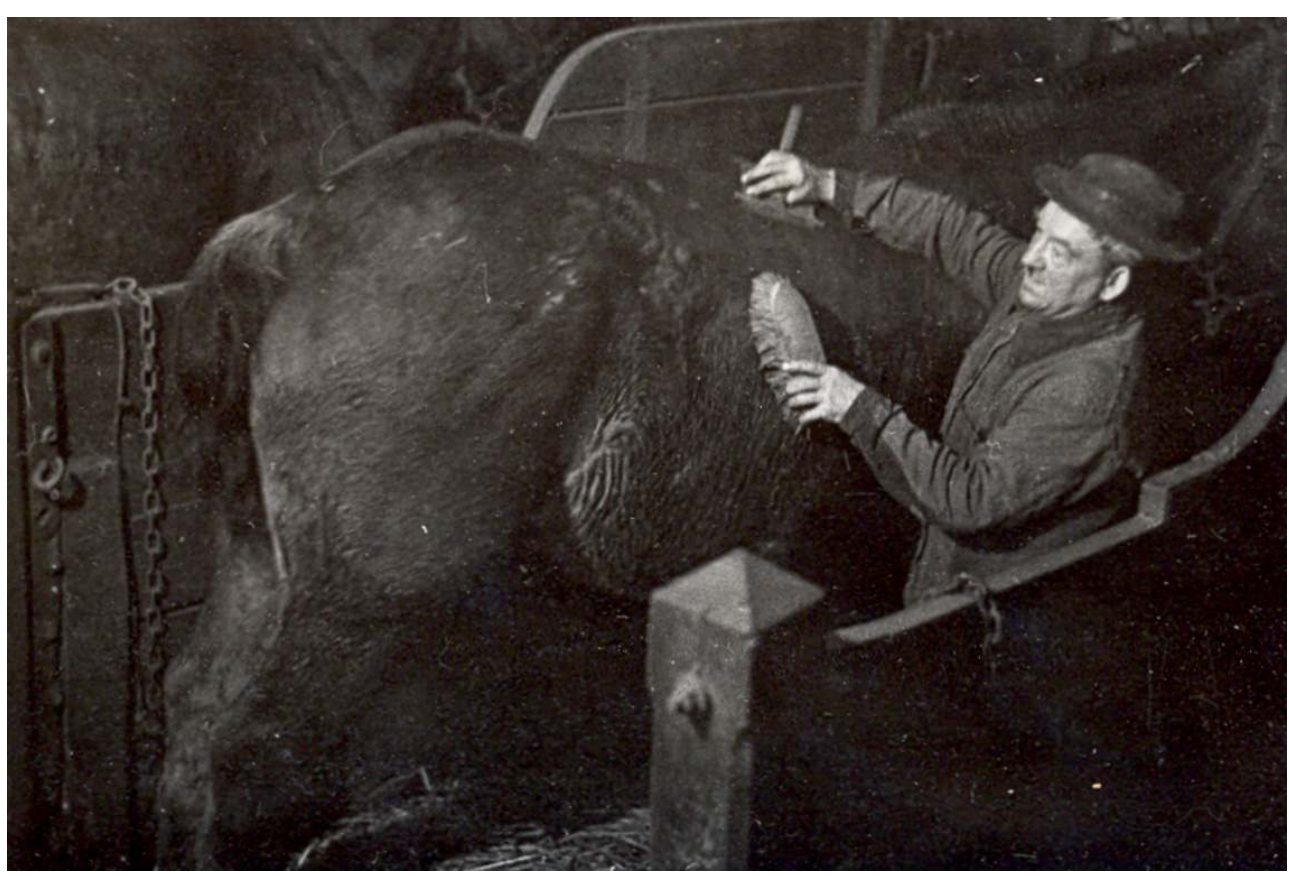

Palefrenier brossant un cheval dans sa stalle au fond, [1937-1945].

(c) ANMT, Charbonnages de France, 20070084054.

\section{Conclusion}

40 L'objectif de cette intervention est de montrer que les archives d'entreprises participent de façon originale à l'écriture de l'histoire du cheval de travail et constituent des sources de première main. Elles livrent des informations sur l'identité des chevaux (provenance, race, genre, couleur, âge, maladie) et sur leurs activités et leur vie quotidienne (travail, pénibilité, risques, hiérarchie).

Bien que faisant partie d'une domesticité collaboratrice, le cheval est pourtant un acteur à part entière et non plus une silhouette dans le paysage, à tel point qu'il entre rapidement dans l'iconographie de la publicité et des illustrations de revues agricoles et industrielles. Son image fait véritablement vendre.

Par ailleurs, l'apogée du cheval-moteur est paradoxalement marquée par le développement industriel au XIX ${ }^{\mathrm{e}}$ siècle et dans le premier tiers du $\mathrm{XX}^{\mathrm{e}}$ siècle. Puis, le cheval est supplanté progressivement par les machines, le train et l'automobile. Il devient vraiment marginal dans l'industrie dans les années 1950 et 1960 en Europe et dans les pays occidentaux.

Les archives d'entreprises sont bien un complément de recherche, qu'elles soient conservées dans le réseau des services publics d'archives (Archives nationales, Archives départementales ou Archives municipales) ou dans les entreprises elles-mêmes. Il faudrait aussi les recouper avec celles d'associations protectrices des animaux et avec celles de l'administration chargée du contrôle sanitaire afin d'apporter des éclairages différents. 
44 , d'autres fonctions du cheval apparaissent aussi fortement dans les fonds des ANMT : le cheval militaire ou policier, le cheval de sport. De même que d'autres équidés interviennent également: poneys, ânes, mulets. Ainsi, tous les aspects de cette domesticité équine laborieuse s'affirment donc dans ces sources.

\section{NOTES}

1. - Gersende Piernas est archiviste et diplômée en histoire des techniques (CDHTE-Cnam, Paris), chargée d'études documentaires aux Archives nationales du monde du travail à Roubaix, où elle est adjointe scientifique de la directrice et responsable des archives d'entreprises et des programmes de numérisation. Gérant aussi les archives du mouvement sportif, elle est membre de la section « archives du sport » (ICA-SPO) du Conseil international des archives. Elle intervient au sein des cours d'archivistique du Master Professionnel « Mémoire et monde du travail » de l'Université Charles-de-Gaulle-Lille III. Elle coopère aussi régulièrement avec la revue h.e. Histoire d'entreprises en publiant des articles qui permettent de mettre en lumière les fonds d'archives des ANMT.

2. - ROCHE, Daniel. La culture de l'Occident, XVI ${ }^{e}$-XIX ${ }^{e}$ siècles. L'ombre du cheval. Tome premier Le cheval moteur. Paris : Fayard, 2008.

3. - GALLAND, Bruno (dir.). Les sources de l'histoire du cheval dans les archives publiques françaises. Paris : Archives nationales, 1993, p. 121.

4. - BOUCHET-BRUN, Ghislaine. Le cheval à Paris de 1850 à 1914. Genève : Droz, 1993 (Mémoires et documents de l'École des Chartes, 37).

5. - Voir «Les maîtres de poste, dont les lignes sont menacées par les chemins de fer, à la Chambre des députés. », du 5 juin 1838, contenu dans le Fonds Dailly, maîtres des postes, 19 AQ 018.

6. - Voir Fonds de la Compagnie générale transatlantique, 9 AQ 35.

7. - Voir les Rapports du Conseil d'administration et de la Commission de comptabilité sur les comptes de l'exercice 1879, 1880, contenus dans le Fonds Dailly, maîtres des postes, 19 AQ 038.

8. - Voir celui de l'année 1878, contenu dans le Fonds Dailly, maîtres des postes, 19 AQ 038.

9. - Voir le Fonds Dailly, 19 AQ 39.

10. - Voir le Fonds René Courtois, 2002070018.

11. - COMBES, Charles. Traité de l'exploitation des mines. Paris : Carilian-Goeury et Victor Dalmont, 1845, Chapitre IX « Travail utile du cheval », p. 17-19.

\section{RÉSUMÉS}

Le cheval de travail est un acteur bien présent du XIX siècle aux années 1960 dans de nombreux fonds d'entreprises conservés aux Archives nationales du monde du travail. Ces documents très variés (correspondance, notes, rapports, listes, livrets, catalogues, plans, photographies) révèlent 
des informations sur l'identité des chevaux, leurs activités et leur vie quotidienne. Le cheval apparaît aussi bien comme un acteur vivant de l'entreprise que comme une partie intégrante du patrimoine matériel de celle-ci. Ayant une activité importante, il est supplanté au fur et à mesure par les machines, le train et l'automobile au cours du XX $\mathrm{XX}^{\mathrm{e}}$ siècle. Ces archives complètent celles conservées dans le réseau des services publics d'archives (Archives nationales, Archives départementales ou Archives municipales) ou dans les entreprises elles-mêmes.

The work horse is a well now, the nineteenth century to the 1960s in many fund companies from the Archives nationales $d u$ monde $d u$ travail à Roubaix. These varied documents (correspondence, memos, reports, lists, booklets, catalogs, maps, photographs) reveal information about the identity of the horses, their activities and their daily lives. The horse appears both as a living actor in the business as an integral part of tangible thereof. Having an important activity, it is replaced as and measurement machines, trains and automobiles in the twentieth century. These records complement those stored in the network of public archives (National Archives, Departmental or municipal Archives) or in the firms themselves.

\section{INDEX}

Mots-clés : cheval de travail, entreprise, mine, carrière, poste, chemin de fer, transport, construction, construction mécanique, brasserie, agriculture, écurie, vétérinaire, maréchalferrant, cocher, postillon, palefrenier, sellerie, bourrellerie, voiture

\section{AUTEUR}

\section{GERSENDE PIERNAS}

archiviste, chargée d'études documentaires aux Archives nationales du monde du travail à Roubaix (ANMT) gersende.piernas@culture.gouv.fr 